\title{
Cohesin components Stag1 and Stag2 differentially influence haematopoietic mesoderm development in zebrafish embryos
}

1 Sarada Ketharnathan ${ }^{1,2}$, Anastasia Labudina ${ }^{1}$, Julia A. Horsfield ${ }^{1,3^{*}}$

$2{ }^{1}$ University of Otago, Department of Pathology, Otago Medical School, Dunedin, New Zealand

$3 \quad{ }^{2}$ Current address: CHEO Research Institute, University of Ottawa, Ottawa, Canada

$4 \quad{ }^{3}$ The University of Auckland, Maurice Wilkins Centre for Molecular Biodiscovery, Private Bag

5 92019, Auckland, New Zealand

6

7 * Correspondence:

8 Julia Horsfield

9 julia.horsfield@otago.ac.nz

10 Keywords: zebrafish, cohesin, haematopoiesis, mesoderm, development. 


\section{Abstract}

13 Cohesin is a multiprotein complex made up of core subunits Smc1, Smc3 and Rad21, and either

14 Stag1 or Stag2. Normal haematopoietic development relies on crucial functions of cohesin in cell

15 division and regulation of gene expression via three-dimensional chromatin organisation. Cohesin

16 subunit STAG2 is frequently mutated in myeloid malignancies, but the individual contributions of

17 Stag variants to haematopoiesis or malignancy are not fully understood. Zebrafish have four Stag

18 paralogues (Stag1a, Stag1b, Stag2a and Stag2b), allowing detailed genetic dissection of the contribution of Stag1-cohesin and Stag2-cohesin to development. Here we characterize for the first time the expression patterns and functions of zebrafish stag genes during embryogenesis. Using lossof-function CRISPR-Cas9 zebrafish mutants, we show that stagla and stag $2 b$ contribute to primitive embryonic haematopoiesis. Both stagla and stag $2 b$ mutants present with erythropenia by 24 hours post-fertilisation. Homozygous loss of either paralog alters the number of haematopoietic/vascular progenitors in the lateral plate mesoderm. The lateral plate mesoderm zone of $s c l$-positive cells is expanded in stagla mutants with concomitant loss of kidney progenitors, and the number of spilpositive cells are increased, consistent with skewing toward primitive myelopoiesis. In contrast, stag $2 b$ mutants have reduced haematopoietic/vascular mesoderm and downregulation of primitive erythropoiesis. Our results suggest that Stag1 and Stag2 proteins cooperate to balance the production of primitive haematopoietic/vascular progenitors from mesoderm. 
Cohesin is a large multi-subunit protein complex that was originally characterised for its role in sister chromatid cohesion during mitosis (Losada, 2008; Onn et al., 2008; Nasmyth and Haering, 2009). Cohesin subunits Smc1A, Smc3 and Rad21 form a large ring-shaped structure that entraps and holds together DNA strands (Nasmyth, 2011). A fourth subunit of either Stag1 or Stag2 binds to cohesin by contacting Rad21 and Smc subunits (Shi et al., 2020), and is required for the association of cohesin with DNA.

Additional roles for cohesin include DNA damage repair and the control of gene expression (Dorsett and Strom, 2012). The gene expression function of cohesin is thought to derive from cohesin's role in three-dimensional genome organisation (Bonora et al., 2014; Rowley and Corces, 2018). Together with the zinc finger protein, CCCTC-binding factor (CTCF), cohesin organizes the genome into large loops known as topologically-associating domains (TADs) (Vietri Rudan and Hadjur, 2015; Hnisz et al., 2016; Rowley and Corces, 2018). The current theory is that cohesin forms loops by extrusion of DNA through the cohesin ring, and CTCF bound in convergent orientation limits extrusion to delineate loop size (Fudenberg et al., 2018; Hansen, 2020).

Inside TADs, cohesin can mediate smaller loops that link genes to their regulatory elements (Merkenschlager and Odom, 2013). Differential formation of sub-TAD gene regulatory loops is thought to be key to cell type specification during development (Hnisz et al., 2016). Several previous studies have linked mutations in cohesin subunits with tissue-specific changes in gene expression (Dorsett, 2009; Merkenschlager, 2010; Horsfield et al., 2012; Kawauchi et al., 2016). Therefore, via its role in genome organization, cohesin plays a crucial role in developmental gene expression.

Germline mutations in genes encoding the cohesin loader NIPBL, or in cohesin subunits, cause a spectrum of human developmental disorders, the best known of which is Cornelia de Lange Syndrome (CdLS). These disorders, known as "cohesinopathies", are characterized by multifactorial developmental anomalies, intellectual disability and growth delay (Liu and Krantz, 2009). On the other hand, somatic mutations in cohesin subunits contribute to the development of several types of cancer, including bladder cancer (15-40\%), endometrial cancer (19\%), glioblastoma (7\%), Ewing's sarcoma (16-22\%) and myeloid leukemias (5-53\%) (De Koninck and Losada, 2016; Hill et al., 2016; Waldman, 2020). How pathogenicity arises from cohesin mutation is poorly understood, but for both cohesinopathies and cancers, causality is thought to derive primarily from the gene expression function of cohesin rather than its cell division role (Hill et al., 2016; Waldman, 2020).

Notably, there is a particularly high frequency of cohesin gene mutations in myeloid malignancies (Kon et al., 2013; Yoshida et al., 2013; Leeke et al., 2014; Thol et al., 2014; Thota et al., 2014; Papaemmanuil et al., 2016). The high frequency of cohesin mutations in myeloid cancers likely reflects cohesin's role in determining haematopoietic lineage identity and controlling the differentiation of haematopoietic stem cells (Mazumdar et al., 2015; Mullenders et al., 2015; Viny et al., 2015; Galeev et al., 2016; Viny et al., 2019).

Several previous studies have investigated the role of cohesin in animal development. In Drosophila, Nipped-B and cohesin control cut gene expression in the wing margin (Dorsett et al., 2005) and mutations in Nipped-B or cohesin genes have dosage-dependent effects on the expression of developmental genes (Dorsett, 2009; Gause et al., 2010). In mice, deficiency in Nipbl or cohesin subunits results in multifactorial developmental abnormalities that mimic CdLS (Kawauchi et al., 
Nipbl and cohesin are important for tissue-specific gene regulation (Monnich et al., 2009; Rhodes et al., 2010; Muto et al., 2011), including expression of hox genes (Muto et al., 2014) and runx genes (Horsfield et al., 2007).

Although animal models have been crucial to understanding the developmental origins of both cohesinopathies (Kawauchi et al., 2016) and haematological malignancies (Viny and Levine, 2018), much remains to be discovered. It is still unclear how cohesin contributes to cell type specification in early development and cell lineage specification. Furthermore, whether all the protein components of cohesin operate equivalently in development is undetermined.

In zebrafish, a forward genetic screen determined that mutation in cohesin subunit rad21 led to loss of runx 1 expression in the posterior lateral plate mesoderm of zebrafish embryos during early somitogenesis. Knock down of the Smc3 subunit of cohesin also eliminated mesoderm runx 1 expression (Horsfield et al., 2007). Runx1 is essential for definitive haematopoiesis, and is itself affected by mutations and translocations in myeloid malignancies (Downing et al., 2000; Speck, 2001). Previous research shows that runxl is directly regulated by Rad21-cohesin in zebrafish (Horsfield et al., 2007; Marsman et al., 2014) and leukaemia cell lines (Antony et al., 2020). Loss of mesoderm-expressed runx 1 at the very earliest time of blood development in rad21 mutants suggests that the onset of haematopoietic differentiation from the mesoderm might require functional cohesin.

The Stag subunits differ from core cohesin subunits Rad21, Smc1 and Smc3 in that they have redundant roles in cell division, such that a complete loss of Stag2 is tolerated due to partial compensation by Stag1. In addition, Stag1 preferentially associates with CTCF to organise TADs whereas Stag2 mediates short-range cell-specific interactions (van der Lelij et al., 2017; Liu et al., 2018; Cuadrado and Losada, 2020). In mice, homozygous loss of Stag1 is lethal at embryonic day 11.5 (E11.5) (Remeseiro et al., 2012a). While adult loss of Stag2 is tolerated, homozygous Stag2-null mouse embryos die by mid-gestation with developmental delay and defective heart morphogenesis (De Koninck et al., 2020). When Stag2 is ablated somatically in adults, increased self-renewal of HSCs accompanied by myeloid skewing is observed (Viny et al., 2019; De Koninck et al., 2020). However, early lethality of Stag mutations makes investigating the embryonic function of Stag1/Stag2 cohesin difficult in mammalian models.

In this study, we characterised the expression of Stag paralogues in early zebrafish development, and investigated whether, like Rad21, cohesin Stag subunits affect haematopoietic differentiation from mesoderm in zebrafish embryos.

\section{RESULTS}

\section{Evolution and embryonic expression of zebrafish Stag paralogues}

Zebrafish have four gene paralogues encoding Stag proteins: stagla, staglb, stag2a and stag2b. To determine if these paralogues are likely to be functional, we characterised their evolutionary conservation and expression in zebrafish embryos.

Phylogenetic analysis of Stag protein sequences using the PhyML algorithm segregated Stag1 and Stag2 into distinct clusters. Stag2b clustered more closely with other vertebrate Stags than Stag2a, while the two Stag1 paralogues had similar levels of divergence (Figure 1A). Whole-mount in situ 
114 hybridisation (WISH) (Figure 1B) and quantitative RT-PCR (qPCR) (Figure 1C,D) was then used to 115 analyse expression of the four stag paralogues in zebrafish embryogenesis.

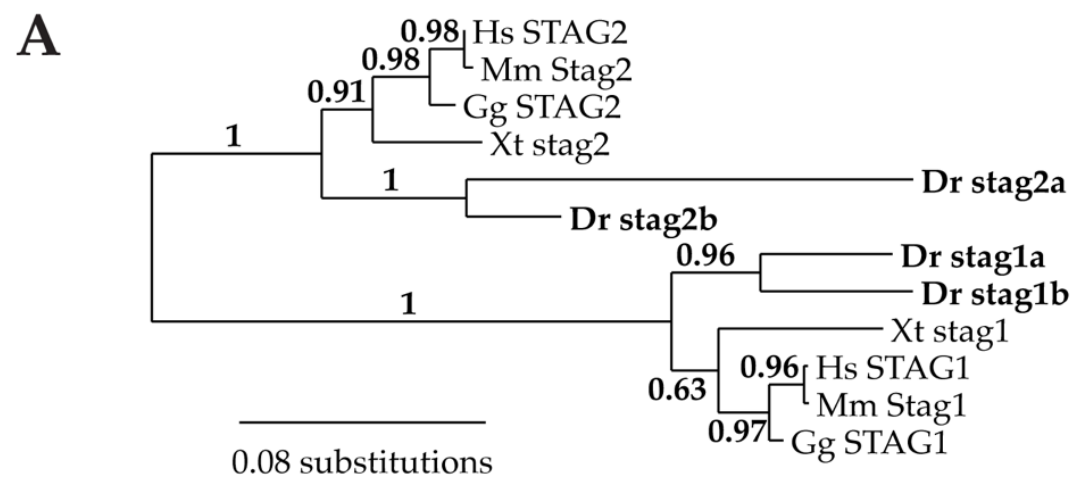

\section{B}

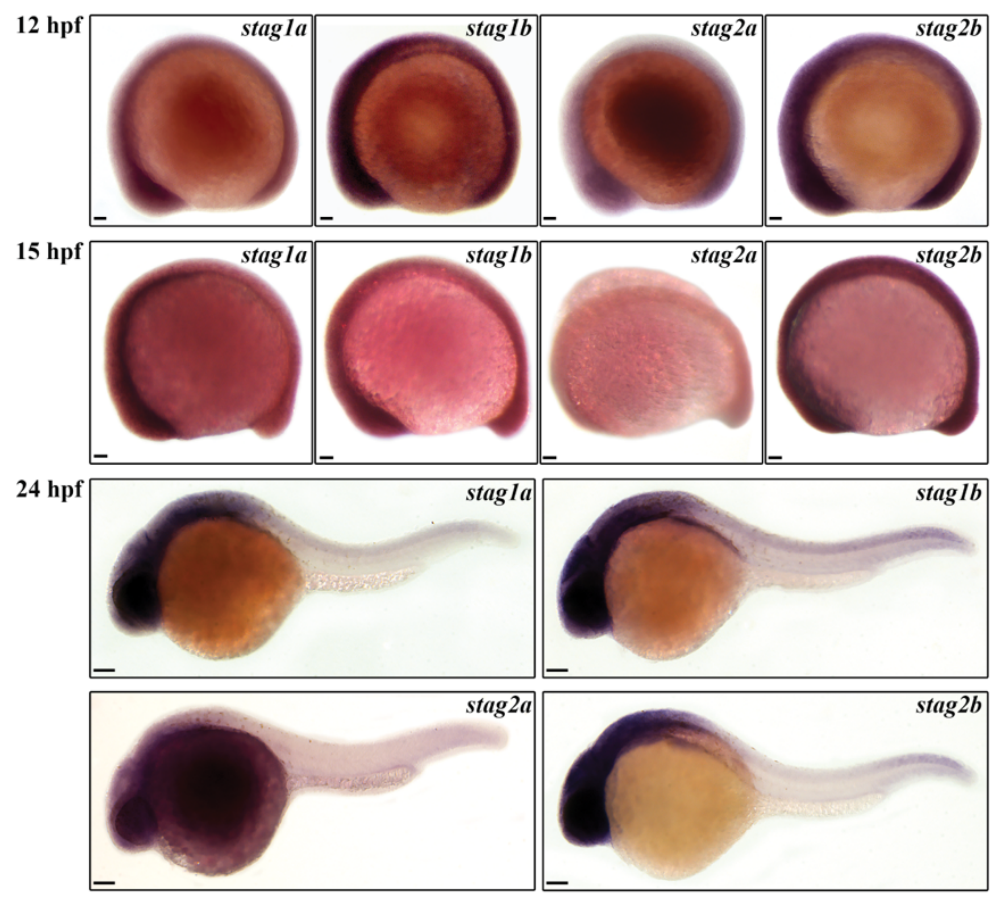

C
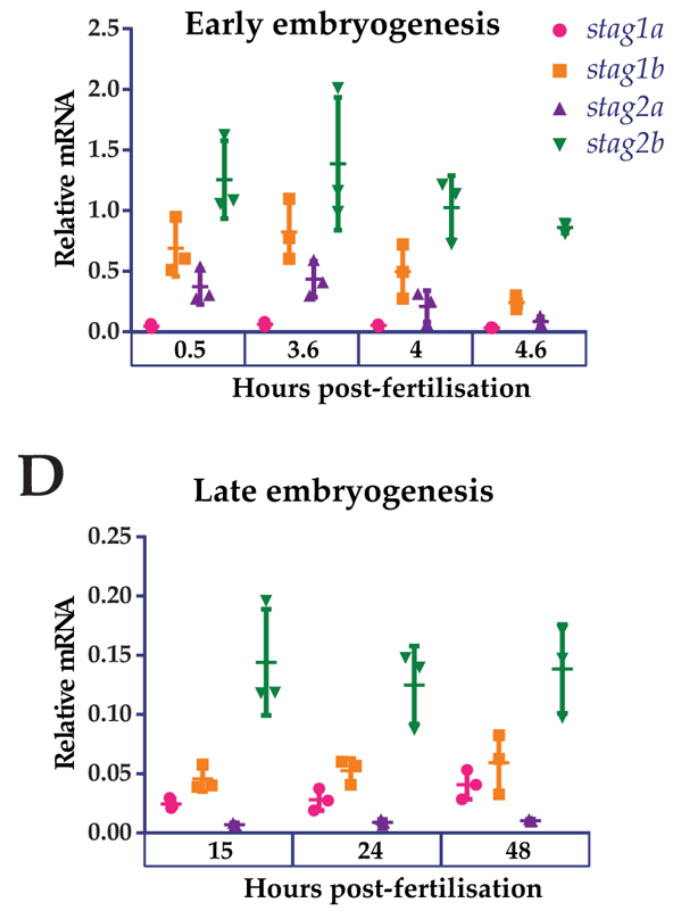

Figure 1. Phylogenetic analysis and embryonic expression of Stag paralogues. (A) Phylogenetic analysis of predicted protein sequences using the maximum likelihood approach. The accession numbers for the protein sequences used in this analysis are listed in Supplementary Table 1. (B) Whole-mount in situ hybridisation of stagla, stag $1 b$, stag2a and stag $2 b$ during early embryogenesis. Lateral views are shown, anterior to the left. Scale bars are $50 \mu \mathrm{m}$ for embryos at $12 \mathrm{hpf}$ and $15 \mathrm{hpf}$ and $100 \mu \mathrm{m}$ for embryos at 24 hpf. (C,D) mRNA expression of stag paralogues at the indicated time points during (C) early embryogenesis and (D) late embryogenesis. Each data point represents mRNA isolated from a pool of 30 embryos. Graphs are mean $+/$ - one standard deviation. Expression was normalised to the reference genes, $b$-actin and $r p l 13 a$ (Supplementary Figure 1A)

116 At early gastrula stages, all four stag paralogues showed ubiquitous expression although stag2a 117 expression was noticeably reduced compared with $s t a g l a / b$ and stag $2 b$. By 24 hours post-fertilisation 118 (hpf), expression of stagla/b and $\operatorname{stag} 2 b$ was robust in anterior regions with high cellular density, 
119 similar to that observed for genes encoding other cohesin subunits (Monnich et al., 2009), while

120 stag2a was barely expressed above background (Figure 1B).

121 We used qPCR to quantify mRNA expression of the stag paralogues at different embryonic

122 timepoints. All four paralogues were both maternally deposited and zygotically expressed with

123 stag $1 b$ and stag $2 b$ being the most expressed throughout embryogenesis. Notably, stagla was

124 predominantly zygotically expressed whereas stag $2 a$ showed maternal deposition that was

125 downregulated post-midblastula transition (Figure 1C,D).

126 In summary, all four Stag paralogues are expressed during development, indicating that they have 127 potential to be functional.

\section{Generation of stag1 and stag2 mutant zebrafish lines}

129 To determine the physiological roles of the four paralogues, we generated loss-of function germline zebrafish mutants in individual stag genes. CRISPR guide RNAs (Supplementary Table 2) were designed to truncate the Stag paralogues upstream of the STAG domain, which spans exons 6 and 7 in all paralogues. We recovered the following germline mutations: $38 \mathrm{bp}$ insertion in exon 3 of stagla, 13 bp deletion in exon 3 of stag $1 b$ and 7 bp deletion in exon 3 of stag $2 b$ (Figure 2A and Supplementary Figure 2). No germline mutations could be recovered in stag $2 a$ despite evaluating multiple guide RNAs. The three zebrafish stag mutant alleles we successfully generated were named stagla ${ }^{n z 204}$, stagl $1 b^{n z 205}$, and $\operatorname{stag} 2 b^{n z 207}$.

To confirm knockdown and to evaluate paralogue compensation, we measured the mRNA levels of the four paralogues at $48 \mathrm{hpf}$ using qPCR (Figure 2B). In stagla ${ }^{\text {nz204 }}$ mutants, stagla mRNA was significantly reduced and was accompanied by significant downregulation of $\operatorname{stag} 1 b$ and $\operatorname{stag} 2 b$ mRNA. In stag $1 b^{n z 205}$ mutants, staglb mRNA was significantly reduced and was accompanied by a significant upregulation of stagla and stag2a mRNA levels, indicating potential transcriptional compensation. In stag $2 b^{n z 207}$ mutants, stag $2 b$ mRNA was modestly but significantly reduced with no changes in the other paralogues.

144 The $\operatorname{stag} 1 a^{n z 204}$, stag $1 b^{n z 205}$, and $\operatorname{stag} 2 b^{n z 207}$ zebrafish mutants were all homozygous viable to adulthood, and fertile. While stagl $a^{n z 204}$ mutants had no apparent larval phenotype, both $\operatorname{stag} 1 b^{n z 205}$ and stag $2 b^{n z 207}$ mutants exhibited mild developmental delay. In addition, stag $2 b^{n z 207}$ mutants had displaced pigment cells in the tail fin by $54 \mathrm{hpf}$ with a penetrance of $\sim 80-85 \%$ (Figure $2 \mathrm{C}$ ). Injection of $200 \mathrm{pg}$ functional stag $2 b$ mRNA in $\operatorname{stag} 2 b^{n z 207}$ mutants rescued the displaced pigment cells (Supplementary Figure 3A).

150 Despite the presence of a 7 bp deletion in the $s t a g 2 b$ gene in $\operatorname{stag} 2 b^{n z 207}$ mutants, downregulation of the stag $2 b$ transcript was rather modest (Figure 2B). Therefore, we sought to confirm loss of function in stag $2 b^{n z 207}$ mutants by determining if a morpholino oligonucleotide targeting stag $2 b$ phenocopies the $\operatorname{stag} 2 b^{n z 207}$ mutation. Injection of $0.5 \mathrm{mM}$ stag $2 b$ morpholino generated the same pigment cell displacement phenotype that was observed in the stag $2 b^{n z 207}$ mutant with no observable toxicity. Furthermore, injection of $0.5 \mathrm{mM}$ stag $2 b$ morpholino into stag $2 b^{n z 207}$ embryos caused no additional abnormalities (Supplementary Figure 3B). These observations indicate that the $\operatorname{stag} 2 b^{n z 207}$ allele is likely to be a true loss of function.

158 Overall, it appears that three of the Stag paralogues, Stag1a, Stag1b and Stag2b, are individually 159 dispensable for zebrafish development and reproduction. We were not able to recover zebrafish 
A

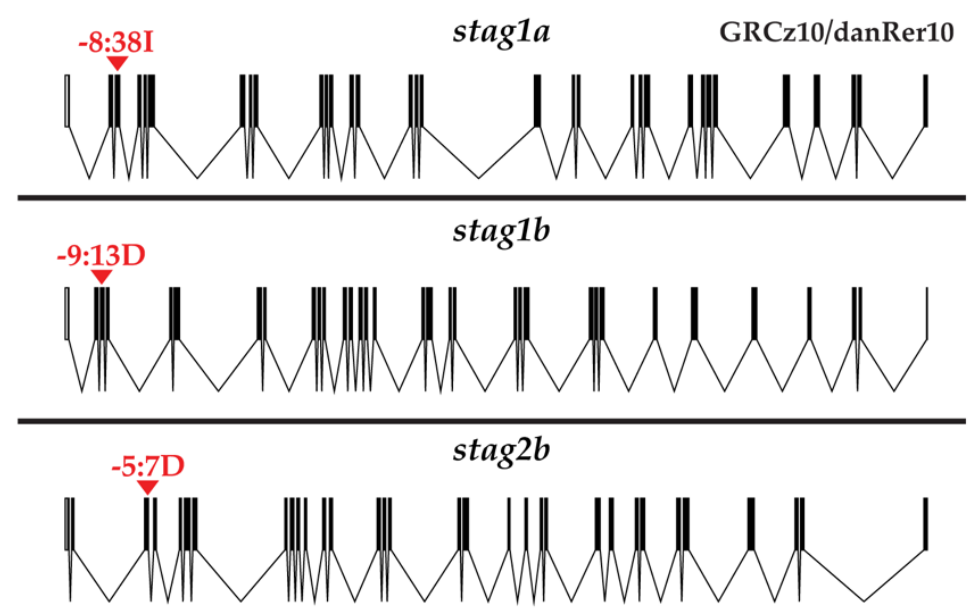

B

stag1a mRNA at $48 \mathrm{hpf}$
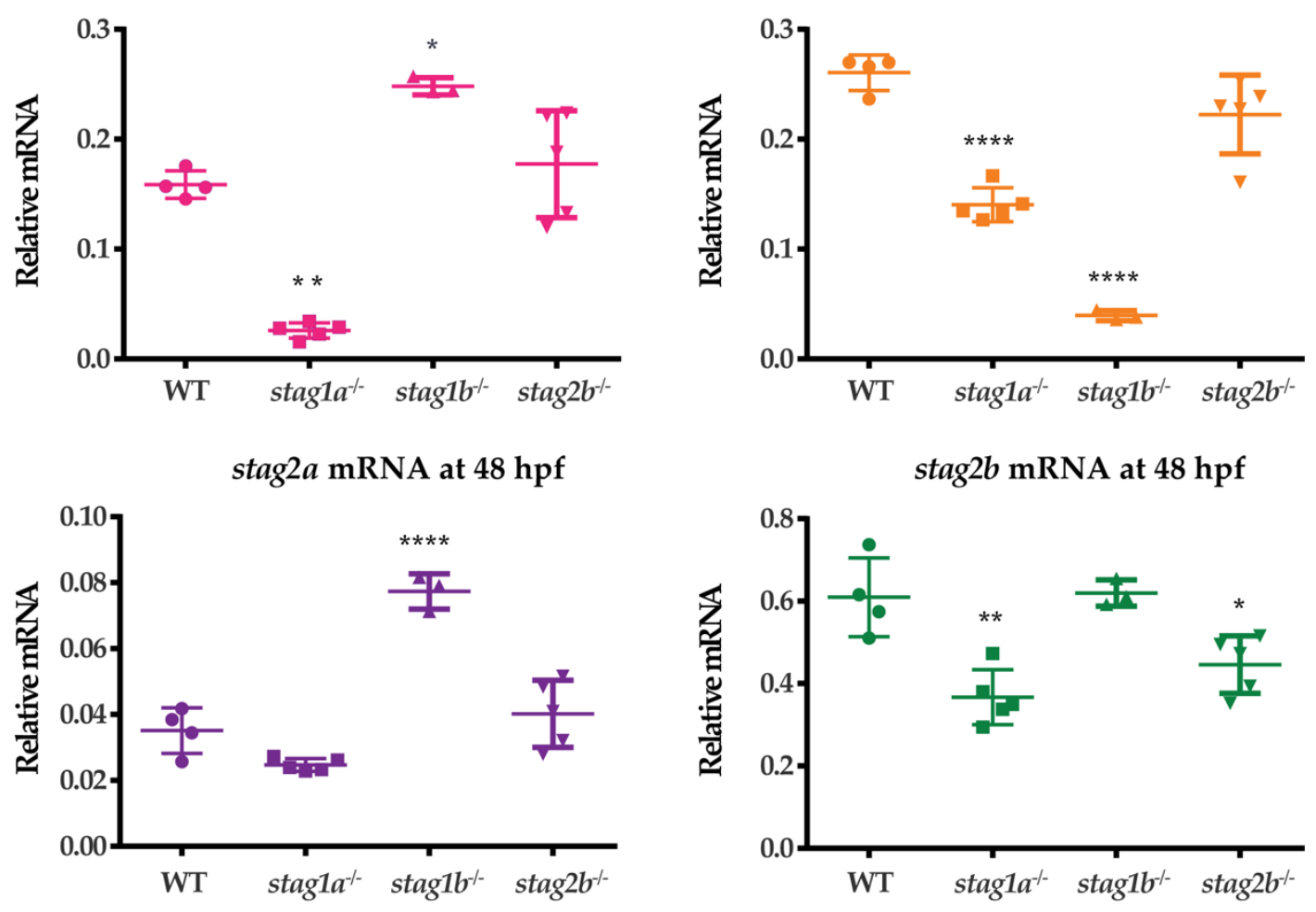

C

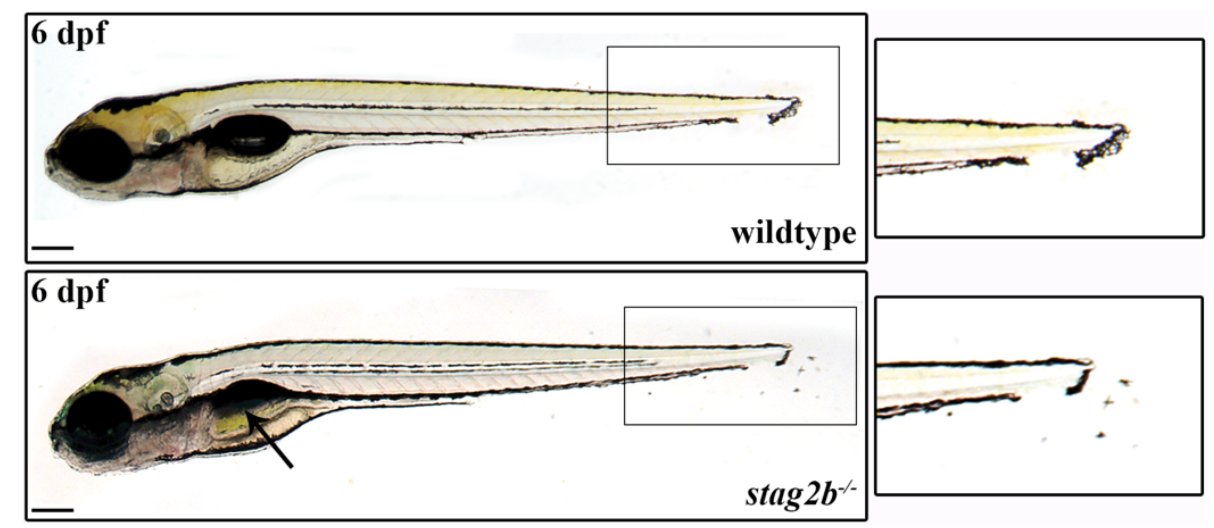

161 Figure 2 legend on following page 
Figure 2. Generation of zebrafish stag germline mutants. CRISPR-Cas9 genome editing was used to generate germline mutations in stagla, staglb and stag2b. (A) Exon diagrams of the respective paralogues showing details of the editing strategy. sgRNA binding sites are marked by red arrowheads with the type of mutation generated indicated above. (B) mRNA levels of the four paralogues in each of the mutant lines indicated on the $x$-axis. Each data point represents mRNA isolated from a pool of 30 embryos. All graphs are mean $+/$ - one standard deviation. * $\mathrm{P} \leq 0.05, * * \mathrm{P} \leq 0.01, * * * \mathrm{P} \leq 0.001, * * * * \mathrm{P} \leq 0.0001$; one-way ANOVA. Expression was normalised to the reference gene, b-actin (Supplementary Figure 1C) (C) stag $2 b^{n z 207}$ mutants have displaced pigment cells in the tail fin, zoomins are shown in insets. Mutants also show mild developmental delay with late swim bladder inflation as indicated by the black arrow. Scale bars are $200 \mu \mathrm{m}$.

162 mutant for $\operatorname{stag} 2 a$; its early maternal expression supports the idea that this subunit may be essential in 163 the germline.

\section{Stag mutations reduce primitive erythroid cells in $\mathbf{2 4}$ hpf zebrafish embryos}

165 Previously, we found that a nonsense mutation in cohesin subunit rad21 inhibits primitive erythropoiesis and blocks the emergence of differentiated myeloid cells (Horsfield et al., 2007). Therefore, we were interested to determine if cohesin stag subunit mutations also affect haematopoiesis in zebrafish embryos. Whole mount in situ hybridisation (WISH) was used to determine if expression of markers of primitive and definitive haematopoiesis is affected in zebrafish stag mutants at $24 \mathrm{hpf}$ and $36 \mathrm{hpf}$. We found that $\operatorname{stag} 1 b^{n z 205}$ mutants had no haematopoietic phenotype (data not shown), but that the stag $1 a^{n z 204}$ and $\operatorname{stag} 2 b^{n z 207}$ mutations both had modest effects on embryonic haematopoiesis.

Expression of gatal marks primitive erythroid cells, and expression of spil (also known as pu.1), primitive myelopoiesis. Expression of gatal at $24 \mathrm{hpf}$ was downregulated in $\operatorname{stagla~}^{n z 204}$ and stag $2 b^{n z 207}$ homozygous and heterozygous mutants, indicating loss of primitive erythroid cells. gatal expression was rescued by injection of functional stagla or stag $2 b$ mRNA (Figure 3A). In contrast, we found that stagla and stag $2 b$ mutation had divergent effects on primitive myelopoiesis: stagla ${ }^{n z 204}$ increased spil expression, while stag $2 b^{n z 207}$ had no effect (Figure 3B). The results suggest that Stag1a and Stag2b promote gatal-mediated primitive erythropoiesis and in addition, Stag1a restricts spil-mediated primitive myelopoiesis.

181 Definitive haematopoietic stem cells (HSCs) in the ventral wall of the dorsal aorta are marked by runx 1 and $c m y b$ expression at 36 hpf. HSC expression of runx 1 was moderately reduced in stagla ${ }^{n 2204}$ mutants and unchanged in stag2 $b^{n z 207}$ mutants (Supplementary Figure 4). Quantitative PCR of RNA isolated from 48 hpf stagla $a^{n z 204}$ and stag $2 b^{n z 207}$ embryos showed that transcript levels of $c m y b, m p x$ and $l y z$ mRNA were similar between mutants and wild type, indicating that definitive myelopoiesis is intact in the mutants. In contrast, gatal expression remained reduced in both stagl $a^{n z 204}$ and stag $2 b^{n z 207}$ mutants at $48 \mathrm{hpf}$ (Figure 3C), indicating that the deficiency in erythropoiesis is sustained from early development. Therefore, Stag1a and Stag2b appear to promote erythropoiesis during embryonic haematopoiesis, but are dispensable for myelopoiesis.

\section{Stag1a and Stag2b are important for specification of $s c l$-positive cells in the haematopoietic mesoderm}


bioRxiv preprint doi: https://doi.org/10.1101/2020.10.19.346122; this version posted October 19,2020 . The copyright holder for this preprint (which was not certified by peer review) is the author/funder, who has granted bioRxiv a license to display the preprint in perpetuity. It is made

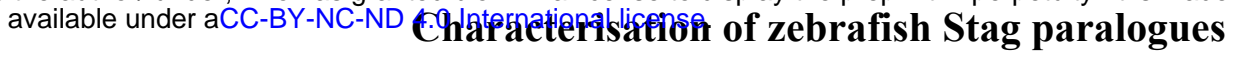

A

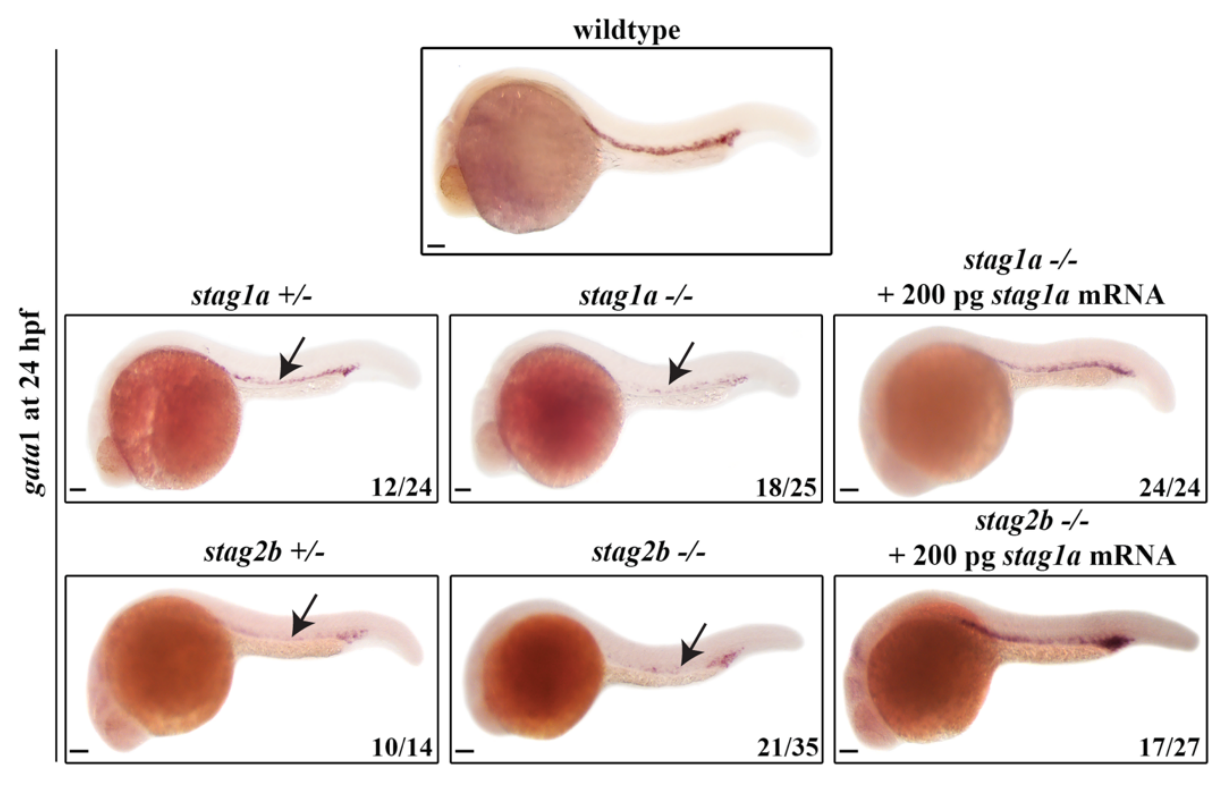

B

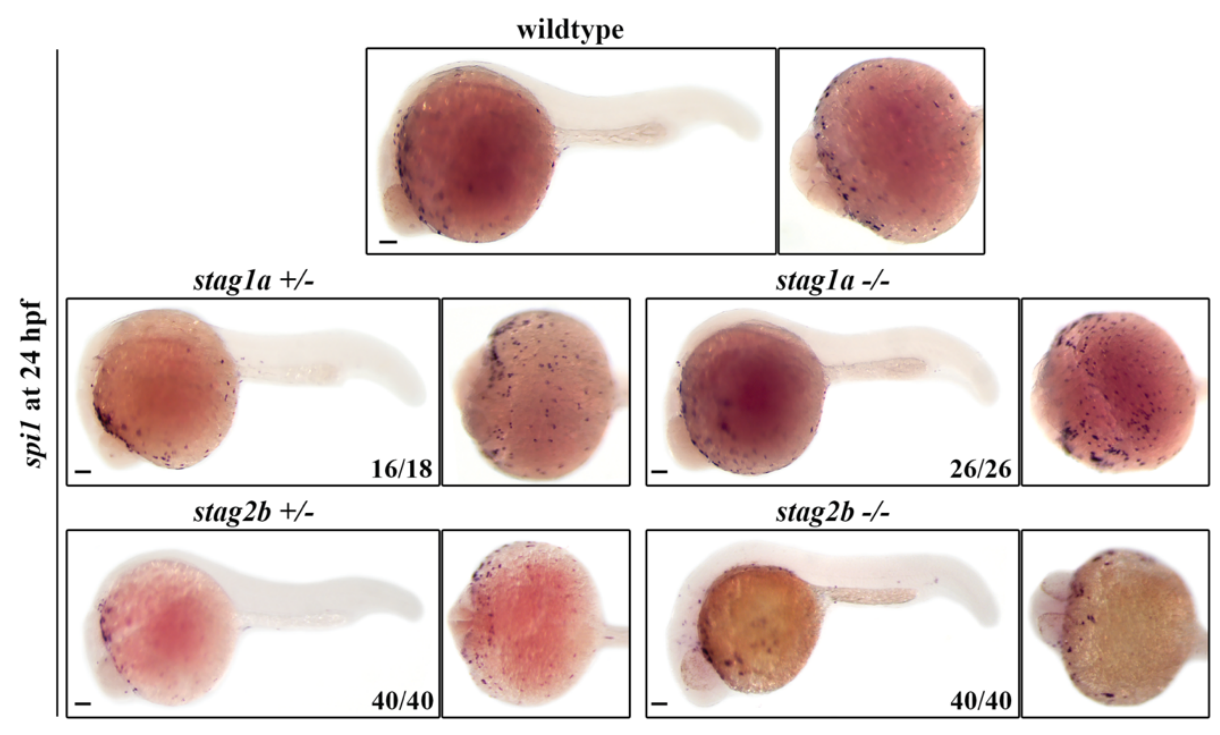

C

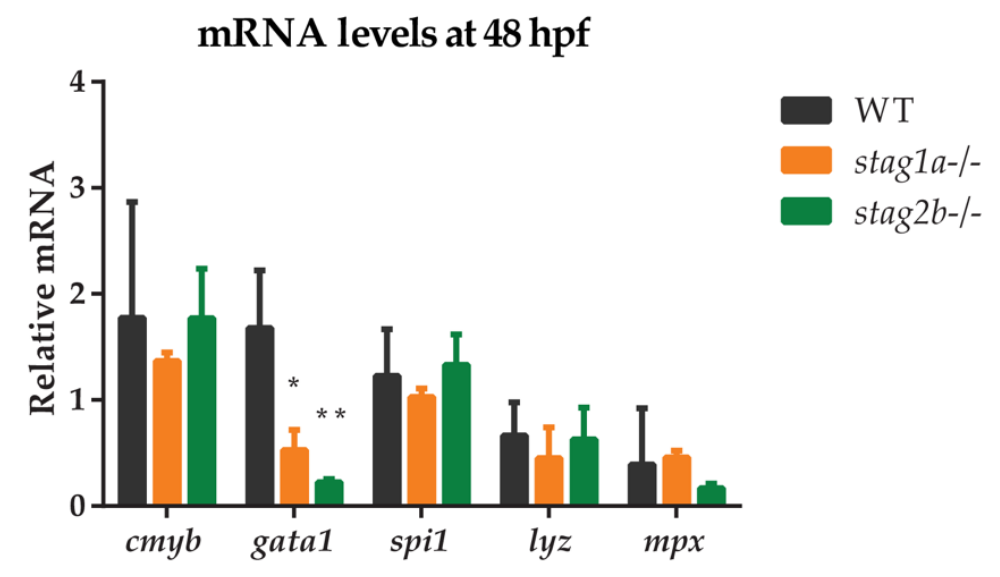

196 Figure 3 legend on following page 
Figure 3. Stag mutations alter the number of gata1- and spi1-positive cells in 24 hpf zebrafish embryos. (A) Lateral views of gatal expression in whole-mount embryos at $24 \mathrm{hpf}$; anterior to the left. gata 1 expression is reduced in stagla $a^{n 2204}+/$ - and stagla ${ }^{n z 204}-/-$ embryos and is rescued upon injection of functional stagla mRNA. gatal expression is reduced in $\operatorname{stag} 2 b^{n z 207}+/-$ and $\operatorname{stag} 2 b^{n z 207}-/-$ embryos and is rescued upon injection of functional stag $2 b$ mRNA. Reduced expression is indicated by arrows. (B) spil expression in whole-mount embryos at $24 \mathrm{hpf}$. Left panels show lateral views and right panels show ventral views; anterior to the left. The number of spi 1 -positive cells is increased in stag $1 a^{n z 204}+/$ - and stag $1 a^{n z 204}-/$ - embryos. spil expression in stag $2 b$ ${ }^{n z 207}$ heterozygous embryos and stag $2 b^{n z 207}$ homozygous mutant embryos is comparable to wildtype. Scale bars are $100 \mu \mathrm{m}$. The number of embryos is indicated in lower-right-hand corners. (C) mRNA levels of haematopoietic stem cell marker $c m y b$ and erythroid or myeloid lineage markers in stagla ${ }^{n z 204}$ and stag $2 b^{n z 207}$ homozygous mutant embryos at $48 \mathrm{hpf}$. The bar graph shows the mean $+/$ - one standard deviation. * $\mathrm{P} \leq 0.05$, $* * \mathrm{P} \leq 0.01$; one-way ANOVA. Expression was normalised to the reference gene, $b$-actin.

observation prompted us to investigate whether stag mutations also affect expression of runx 1 and other lineage-defining genes in the intermediate mesoderm.

WISH with a riboprobe detecting runxl expression in the PLM on $15 \mathrm{hpf}$ embryos (14 somites) revealed that stag $1 a^{n z 204}$ and stag $2 b^{n z 207}$ mutants had relatively normal PLM runxl expression (Figure 4A). We observed minor expansion in the PLM domain of runxl in stag $1 a^{n z 204}$ mutants, and minor localised reduction of runx 1 expression in stag $2 b^{n z 207}$ mutants; however, qPCR revealed that total runxl transcript levels are not significantly different between mutants and wild type (Figure 4C). Therefore, unlike rad21 mutation, stagla or stag $2 b$ mutations are by themselves not sufficient to cause dramatic changes to runxl expression.

Expression of the $s c l$ (tal-1) gene marks a subset of cells in the PLM that will later go on to assume either vascular or haematopoietic identity. Surprisingly, we observed significant differences in the expression pattern of $s c l$ in the PLM of stagl $a^{n z 204}$ and $\operatorname{stag} 2 b^{n z 207}$ mutants at $15 \mathrm{hpf}$ (14 somites) (Figure 4B). An expanded lateral domain of $s c l$ expression appeared in the PLM of $\operatorname{stag} 1 a^{n z 204}$ mutants, and was rescued by injection of stagla mRNA (Figure 4B). In contrast, $s c l$ expression was reduced in the anterior PLM of $\operatorname{stag} 2 b^{n z 207}$ mutants, and this was rescued by injection of $s t a g 2 b$ mRNA (Figure 4B). The observed changes in $s c l$ expression were reinforced by qPCR analysis (Figure 4C), which showed an increase of $s c l$ transcript in $s t a g l a^{n z 204}$ and decrease in $s t a g 2 b^{n z 207}$ mutants, respectively. In contrast to observations in $24 \mathrm{hpf}$ embryos, gatal transcript levels were increased in stagla $a^{n z 204}$ mutants along with a slight increase in spil mRNA. Expression of the vascular marker, fli 1 , was not significantly altered (Figure 4C).

217 The results suggest that during early somitogenesis in stagl $1 a^{n z 204}$ mutants, $s c l$-positive cell numbers

218 are expanded and accompanied by the upregulation of primitive haematopoietic markers. In contrast, both $s c l$ and gatal are downregulated in $s t a g 2 b^{n z 207}$ mutants suggesting a reduction in $s c l$-positive 220 haematopoietic/vascular progenitors.

\section{Loss of Stag1a, but not Stag2b, alters gene expression domains in the posterior lateral} mesoderm

223 During early somitogenesis, the PLM contains non-overlapping stripes of pax $2 a$-expressing

224 pronephric progenitors adjacent to the $s c l$-expressing cells. We were curious to know whether 
A

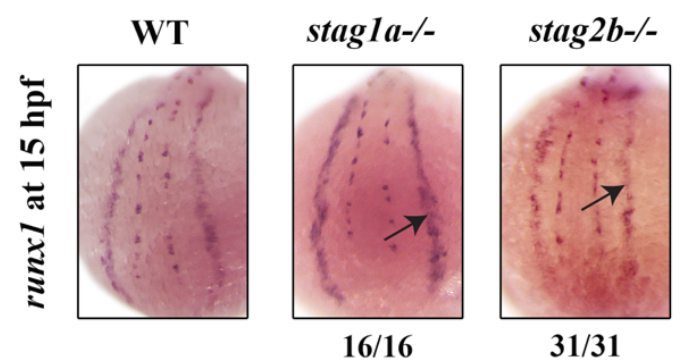

B

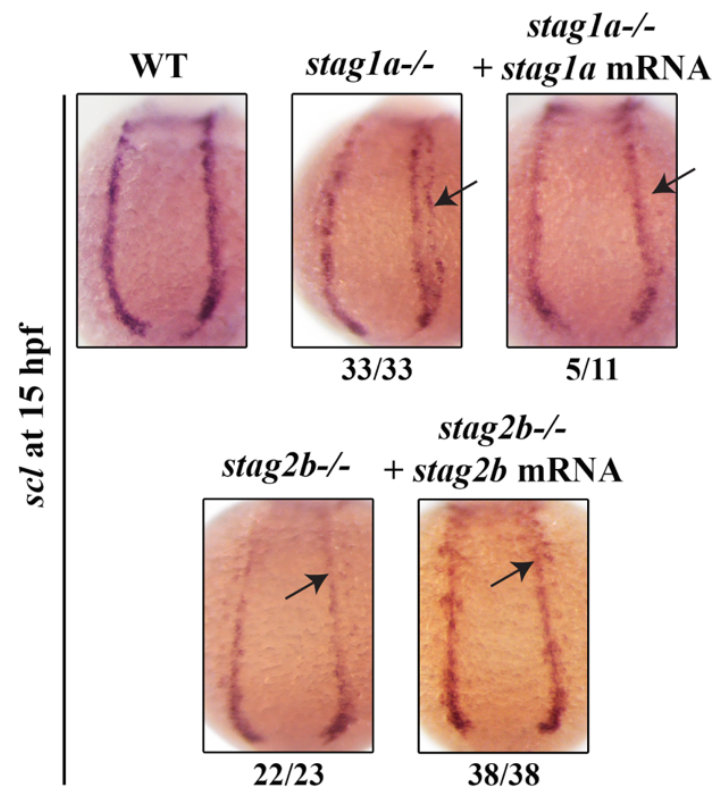

C

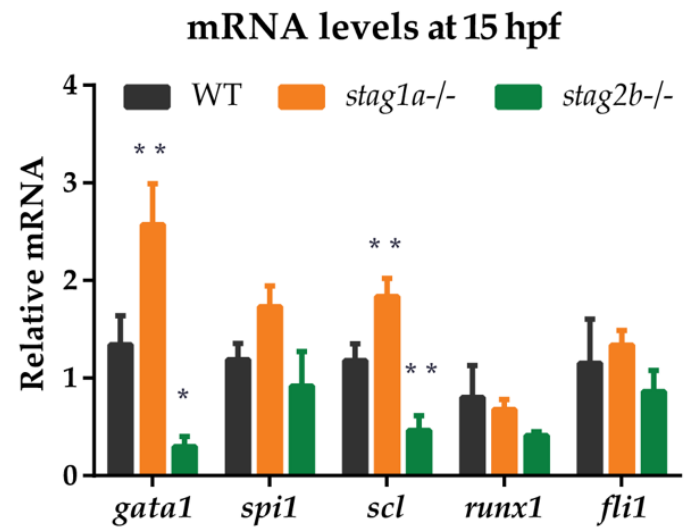

Figure 4. stagla and stag $2 b$ mutations alter the number of $s c l$-positive cells in the posterior lateral mesoderm at 15 hpf. (A) runxl expression in wholemount embryos at $15 \mathrm{hpf}$. Posterior views of the PLM are shown; dorsal to the top. In stagl $1 a^{n z 204}$ homozygous mutant embryos, runxl expression is slightly increased. In stag $2 b^{n z 207}$ homozygous mutant embryos, runx 1 expression is slightly reduced. Changes in expression are marked by arrows and the number of embryos is indicated below each panel. (B) $s c l$ expression in whole-mount embryos at $15 \mathrm{hpf}$. Posterior views of the PLM are shown; dorsal to the top. In stagla $a^{n z 204}$ homozygous mutant embryos, expanded expression of $s c l$ laterally into the PLM is dampened upon injection of functional stagla mRNA. In stag $2 b^{n z 207}$ homozygous mutant embryos, scl expression is reduced in the anterior PLM and is rescued upon injection of functional stag $2 b$ mRNA. Changes in expression are marked by arrows and the number of embryos is indicated below each panel. (C) mRNA levels of mesoderm-derived haematopoietic and endothelial markers at $15 \mathrm{hpf}$. The bar graph shows the mean +/one standard deviation. * $\mathrm{P} \leq 0.05, * * \mathrm{P} \leq 0.01$; oneway ANOVA. Expression was normalised to the reference genes, b-actin and rpll3a (Supplementary Figure 1B).

changes in the $s c l$-positive population in the stag mutants influenced adjacent cell populations, such as pronephric progenitors, in the mesoderm.

At $12 \mathrm{hpf}\left(10\right.$ somites), $s c l$ expression was expanded in stag $1 a^{n z 204}$ mutants, while in stag $2 b^{n z 207}$ mutants, $s c l$ expression was slightly reduced (Figure $5 \mathrm{~A}$ ). This is finding is consistent with observations of 15 hpf embryos (Figure 4B,C; Supplementary Figure 5A). Notably, the PLM zone of pax $2 a$ expression was reduced concomitant with expansion of $s c l$-expressing cells in the PLM of stagla ${ }^{n z 204}$ mutants (Figure 5B; Supplementary Figure 5B). These results suggest that scl-positive haematopoietic/endothelial progenitors are expanded at the expense of pronephric progenitors in stagla $a^{n z 204}$ mutants. In contrast, in $\operatorname{stag} 2 b^{n z 207}$ mutants with reduced $s c l$ transcript, expression of 
234 pax2a was maintained in the PLM but reduced in the optic stalk compared with wild type (Figure

235 5B,C; Supplementary Figure 5B).

A

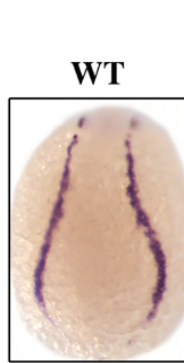

scl at $12 \mathrm{hpf}$

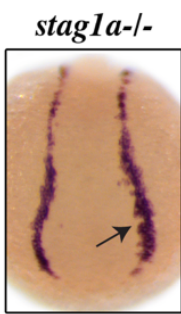

$23 / 24$ stag $2 b-/-$

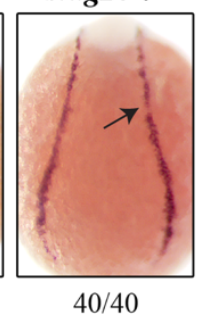

B

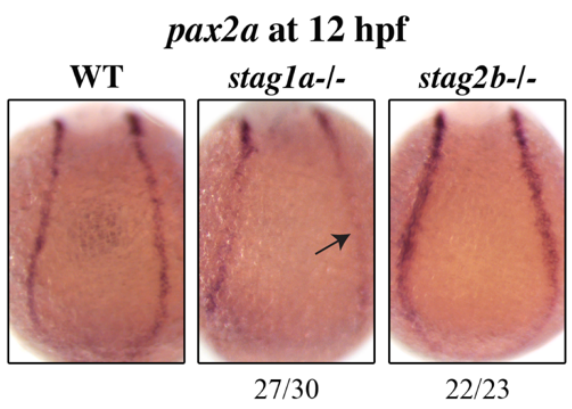

C $\operatorname{pax} 2 a$ at $12 \mathrm{hpf}$
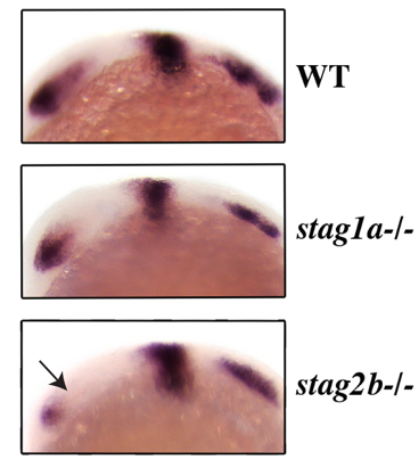

D

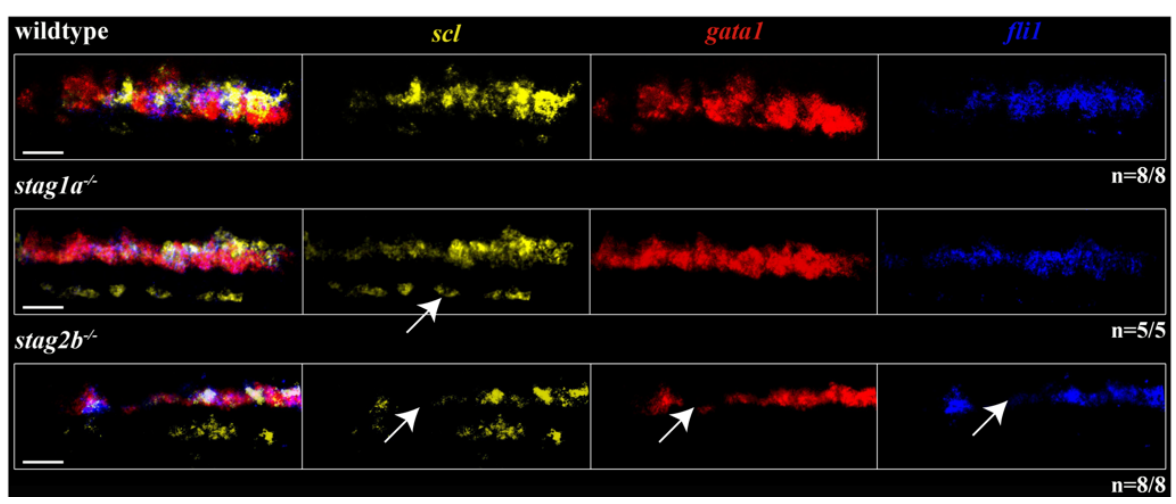

Figure 5. stag1 $a$ and stag $2 b$ mutations affect cell identity in the posterior lateral mesoderm at 12 hpf. (A) $\mathrm{scl}$ expression in whole-mount embryos at $12 \mathrm{hpf}$, posterior views of the PLM; dorsal to the top. In stag $1 a^{n z 204}$ homozygous mutant embryos $s c l$ expression is expanded. In $\operatorname{stag} 2 b^{n z 207}$ homozygous mutant embryos, $s c l$ expression is reduced. Changes in expression are marked by arrows and the number of embryos is indicated below each panel. (B) pax2 expression in whole-mount embryos at $12 \mathrm{hpf}$, posterior views of the PLM; dorsal to the top. In stag $1 a^{n z 204}$ homozygous mutant embryos, pax2 expression in the PLM is markedly reduced. In stag $2 b^{n z 207}$ homozygous mutant embryos, pax 2 expression is comparable to wild type. Changes in expression are marked by arrows and the number of embryos is indicated below each panel. (C) pax2 expression in whole-mount embryos at $12 \mathrm{hpf}$, lateral views of the head region; anterior to the left. Anterior pax2 expression is specifically reduced in the optic stalk of $\operatorname{stag} 2 b^{n z 207}$ homozygous mutant embryos. (D) Multiplexed in situ HCR of $s c l$ (Alexa Fluor 488, false colour yellow), gatal (Alexa Fluor 594, false colour red) and flil (Alexa Fluor 647, false colour blue) expression at 15 hpf. High magnification maximum intensity projections of a single PLM stripe; posterior views with anterior to the left. Expression domains of $s c l$ broadly overlap gatal and flil in all embryos. Ectopic $s c l$ expression, indicated by white arrow, in stagl $1 a^{n 2204}$ homozygous mutant embryos does not overlap gatal or flil expression domains. In stag $2 b^{n z 207}$ homozygous mutant embryos, expression of all three markers is reduced. Scale bars are $10 \mu \mathrm{m}$. The number of embryos analysed is indicated below the respective panels. 
A subset of $s c l$-positive cells also express gatal and acquire a haematopoietic fate while the remaining cells express flil acquiring an endothelial fate. We next wanted to determine whether $s c l$ positive cells are skewed towards a haematopoietic or vascular fate in the stag mutants. Multiplex in situ hybridisation using HCR revealed that the expression of gatal and flil largely overlap that of scl in the PLM (Figure 5D; Supplementary Figure 5A). Ectopic $\mathrm{scl}$ expression seen in stagla $1 a^{n z 204}$ mutants did not overlap gatal or flil expression, but gatal expression appeared more intense than wild type, consistent with qPCR results (Figure 5D; Figure 4C; Supplementary Figure 5A). We detected no differences in the relative composition of $\mathrm{scl}^{+} / \mathrm{gatal}^{+}$and $\mathrm{scl}^{+} / \mathrm{flil}^{+}$cells in the PLM of

A

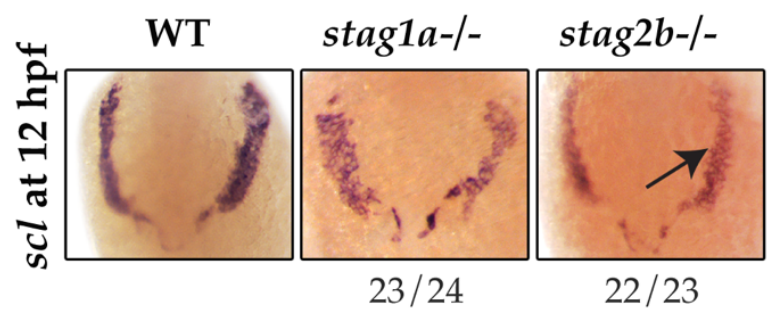

C
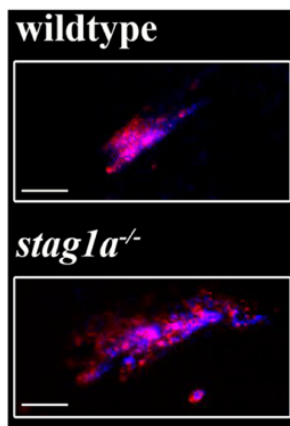

stag $2 b^{-}$

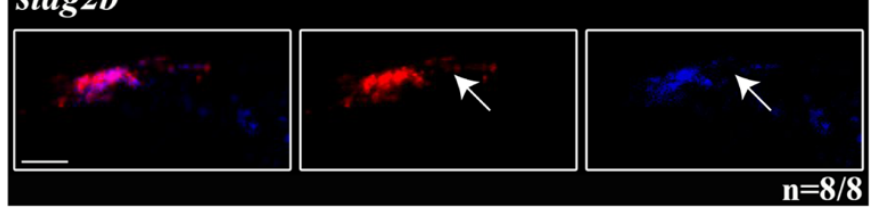

B

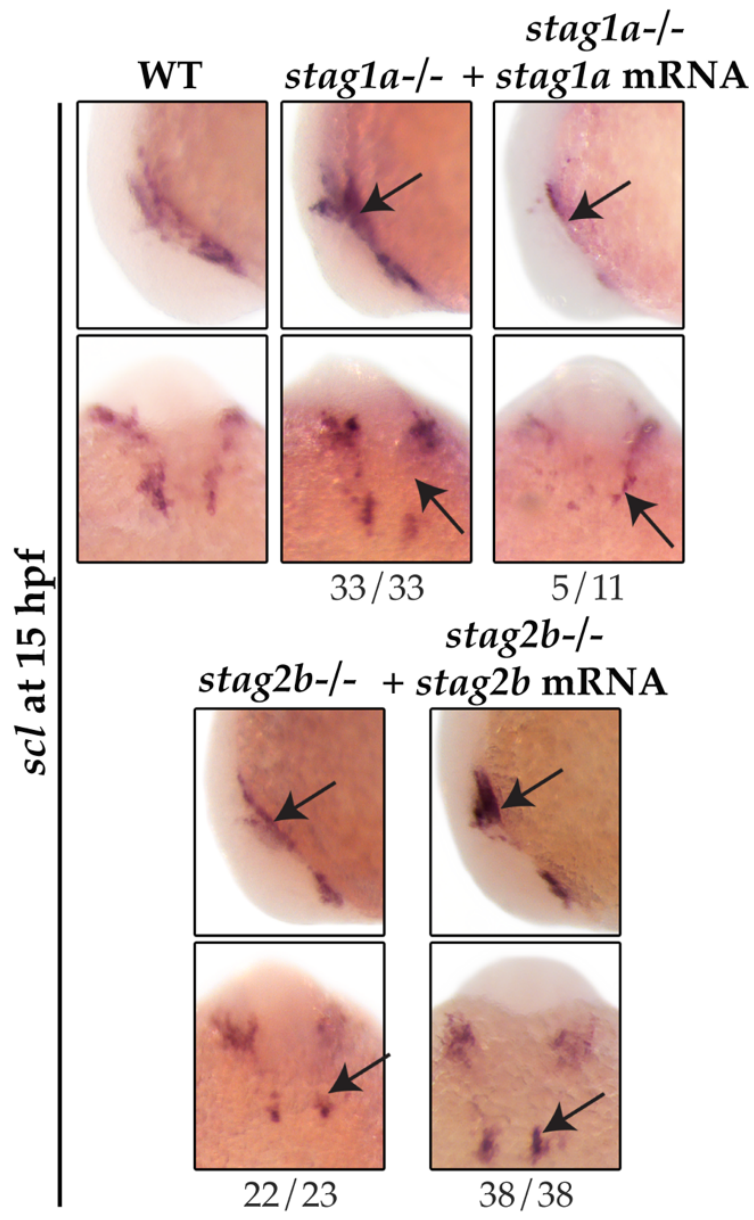

Figure 6. stag1 $a$ and $\operatorname{stag} 2 b$ mutations differentially alter the production of primitive myeloid cells in the anterior lateral mesoderm at $12 \mathrm{hpf}$. (A) $\mathrm{scl}$ expression in whole-mount embryos at $12 \mathrm{hpf}$. Ventral views of ALM are shown; dorsal to the top. scl expression is comparable to wildtype in stagla $a^{n z 204}$ homozygous mutant embryos and reduced in stag $2 b^{n z 207}$ homozygous mutant embryos. (B) $s c l$ expression in whole-mount embryos at $15 \mathrm{hpf}$. Top panels show lateral views and bottom panels show ventral views of the ALM. Expanded $s c l$ expression in the ALM of stag $1 a^{n z 204}$ mutants is rescued upon injection of functional stagla mRNA. The reduced scl expression in the ALM of $s t a g 2 b^{n z 207}$ mutants is rescued upon injection of functional stag $2 b$ mRNA. Changes in expression are marked by arrows and the number of embryos is indicated below each panel. (C) Multiplexed in situ HCR of runxl and spil at 15 hpf. Maximum intensity projections of a single ALM stripe; lateral views with dorsal to the top. Expression domains of runxl (Alexa Fluor 647) and spil (Alexa Fluor 514, false colour blue) broadly overlap in all embryos. Both runx1 and spil are expanded in stagl $a^{n z 204}$ embryos but reduced in stag $2 b^{n z 207}$ embryos. Changes in expression are indicated by white arrows. Scale bars are $10 \mu \mathrm{m}$. The number of embryos analysed is indicated below the respective panels. 
mutants (Supplementary Figure 5A). The results suggest that in stagla ${ }^{n z 204}$ mutants, expanded $s c l$ expression does not appear to skew cell fate in the PLM, but transiently increases gatal expression.

In stag $2 b^{n z 207}$ mutants, the expression domains of $s c l$, gatal and flil was reduced in the PLM (Figure 5D; Supplementary Figure 5A). Cell composition of the PLM was unchanged in stag2 $b^{n z 207}$ mutants (Supplementary Figure 5A), suggesting that reduced scl, gatal and flil does not influence PLM cell fate.

\section{Stag1a or Stag2b loss differentially affects the production of primitive myeloid cells in the anterior lateral mesoderm}

We next asked if stag mutants also affect haematopoietic cell specification in the anterior lateral mesoderm (ALM), a site of primitive myelopoiesis (Berman et al., 2005). At $12 \mathrm{hpf}, \mathrm{scl}$ expression in the rostral blood island marks a population of cells fated to become spil-positive myeloid cells or fli1-positive endothelial cells.

$s c l$ expression was normal in the ALM of stagla $a^{n z 204}$ mutants at $12 \mathrm{hpf}$ (Figure 6A) but by $15 \mathrm{hpf} s c l$ expression was markedly increased in stagla ${ }^{n z 204}$ (Figure 6B, Supplementary Figure 5C). Increased scl expression in the ALM of stagl $1 a^{n z 204}$ mutants was reversed by injection of functional stagla mRNA, which reduced $s c l$ expression to below normal. In stag $2 b^{n z 207}$ mutants, $s c l$ expression was reduced in the ALM at both 12 and $15 \mathrm{hpf}$, and was robustly rescued upon injection of stag $2 b$ mRNA (Figure 5B).

Multiplex HCR expression analysis showed that the population of ALM cells that co-express runx1 and spil are expanded in the ALM of stagla ${ }^{n z 204}$ mutants (Figure 6C). In contrast, the same spil/runx 1-positive ALM population was reduced in $s t a g 2 b^{n z 207}$ mutants. Since there was also a modest increase in spil-positive cells in stagla ${ }^{n z 204}$ mutants at $24 \mathrm{hpf}$ (Figure 3B), these results are consistent with the idea that excess $s c l$ in $\operatorname{stag} 1 a^{n z 204}$ mutants promotes myelopoiesis in the anterior blood island.

Taken together, the results suggest that in early somitogenesis, Stag1a normally restricts $s c l$ expression in the ALM and PLM, such that its loss in stag $1 a^{n z 204}$ mutants results in a modest expansion of primitive erythroid and myeloid cells at the expense of pronephros specification. In contrast, Stag $2 \mathrm{~b}$ positively regulates the number of $s c l$-expressing cells and its loss in $s t a g 2 b^{n z 207}$ mutants leads to a reduction of $s c l$-derived lineages. However, by $24 \mathrm{hpf}$ gatal-positive cells are reduced in both stag $1 a^{n z 204}$ and $\operatorname{stag} 2 b^{n z 207}$ mutants, suggesting that erythropenia is a common consequence of an imbalance in scl-positive cells. Because both stagla ${ }^{n z 204}$ and stag $2 b^{n z 207}$ mutants are homozygous viable, there must be sufficient redundancies and plasticity to overcome these stag mutations in later development.

\section{DISCUSSION}

279 All four Stag paralogues are expressed in early embryogenesis, suggesting that they are likely to have a function in early development. The maternally and zygotically expressed $s t a g 1 b$ and $s t a g 2 b$ are the most abundant of the zebrafish Stags. While zebrafish Stag1a and Stag1b are more or less equally related to mammalian Stag1, the higher zygotic expression of staglb suggests that it is the most predominant isoform in zebrafish. Of the two Stag2 isoforms, stag $2 b$ is the most abundantly 
expressed and is also most closely related to mammalian Stag2, suggesting that Stag2b is likely to be the predominant Stag2 in zebrafish.

The stag2a paralogue mRNA is present in early embryos up until the mid-blastula transition and then is rapidly downregulated. Interestingly, we detected robust stag2a expression in the ovaries of adult zebrafish (Supplementary Figure 6), and little expression elsewhere in adults. It is possible that stag $2 a$ is required in oocytes for development pre-zygotic genome activation, but is dispensable at later stages. Significantly, we were not able to isolate a CRISPR mutant for stag2 $a$, raising the possibility that Stag2a is essential in oocytes its loss does not allow for transmission of a mutation.

All three germline mutations successfully isolated for the Stag paralogues are homozygous viable and fertile, indicating that there is likely to be functional redundancy among Stag proteins throughout development and reproduction. Compensation could be partly transcription based, for example, stag $1 b^{n z 205}$ mutant embryos upregulated expression of stagla and stag2a. Fish that were mutant for the most abundant Stags, stag $1 b^{n z 205}$ and $\operatorname{stag} 2 b^{n z 207}$, exhibited a slight developmental delay as larvae, and had displaced pigment cells in the tail fin. However, only the stagla $a^{n z 204}$ (which had no morphological phenotype) and $\operatorname{stag} 2 b^{n z 207}$ mutants produced haematopoietic phenotypes in embryos younger than $48 \mathrm{hpf}$. The sharp increase of stagla expression and the abrupt downregulation of stag2 $a$ at the mid-blastula transition (leaving stag $2 b$ as virtually the sole zygotic Stag2) might explain why these two particular mutations caused phenotypes in embryos.

Analysis of primitive haematopoiesis from 24-48 hpf showed that both the $\operatorname{stag} 1 a^{n z 204}$ and $\operatorname{stag} 2 b^{n z 207}$ mutants had a profound decrease in erythroid cells. These findings are in partial agreement with data from mice. Somatic removal of Stag2 in mice resulted in increased myeloid progenitors and decreased megakaryocyte-erythrocyte progenitors, with consequential myeloid skewing (Viny et al., 2019; De Koninck et al., 2020). However, there is no haematopoietic phenotype in Stagl-mutant mice (Viny et al., 2019), contrasting with the erythropenia we observed in zebrafish stag $1 a^{n z 204}$ mutants at 24 and $48 \mathrm{hpf}$.

Although stagl $1 a^{n z 204}$ and $\operatorname{stag} 2 b^{n z 207}$ mutants both had erythroid deficiency, unexpectedly, only the stagla ${ }^{n z 204}$ mutant presented with additional early haematopoietic alterations. These included a reduction in runx 1 -positive definitive HSCs at $36 \mathrm{hpf}$ in stagla ${ }^{n z 204}$ mutants, and striking changes to expression of $s c l$ in the PLM at 12 and $15 \mathrm{hpf}$.

313 The basic helix-loop-helix protein Scl/Tal-1 is expressed in mesoderm and marks both vascular and haematopoietic lineages. Scl is thought to program ventral mesoderm to a haematopoietic fate (Orkin, 1995; Davidson and Zon, 2000; Prummel et al., 2020). Overexpression of zebrafish scl leads to an overproduction of blood from mesoderm at the expense of other non-axial mesoderm fates (Gering et al., 1998). Consistent with this, we observed a reduction in expression of pax $2 a$ in the pronephric mesoderm in stagl $1 a^{n z 204}$ mutants that had expanded expression of $s c l$. However, a concomitant increase in expression of downstream haematopoietic markers gatal and spil was only transitory in stagla ${ }^{n z 204}$ mutants. Expression of gatal and spil is increased in 15 hpf stagla ${ }^{n z 204}$ mutants but by 24 hpf, spil expression was normal and gatal expression was reduced.

Stag2 depletion in mice induces both an increase in self-renewal and reduced differentiation capacity 
of Stag2b had little effect on spil expression, but did lead to reduced primitive haematopoiesis overall.

328 The phenotypes of stagl $a^{n z 204}$ and $\operatorname{stag} 2 b^{n z 207}$ mutants have opposite effects on $s c l$ expression in early somitogenesis (12 and $15 \mathrm{hpf}$ ), but a similar reduction in gatal-positive cells by $24 \mathrm{hpf}$. We suggest that loss of Stag2b leading to reduced $s c l$ expression limits the pool of progenitors that can contribute to primitive haematopoiesis. Conversely, we propose that increased $s c l$ expression caused by loss of Stag1a increases haematopoietic progenitors that are subsequently exhausted by early differentiation. These scenarios would explain the erythropenia observed in both $\operatorname{stag} 1 a^{n z 204}$ and stag $2 b^{n z 207}$ mutants 334 by 24 hpf(Figure 7).

\section{Primitive erythropoiesis}

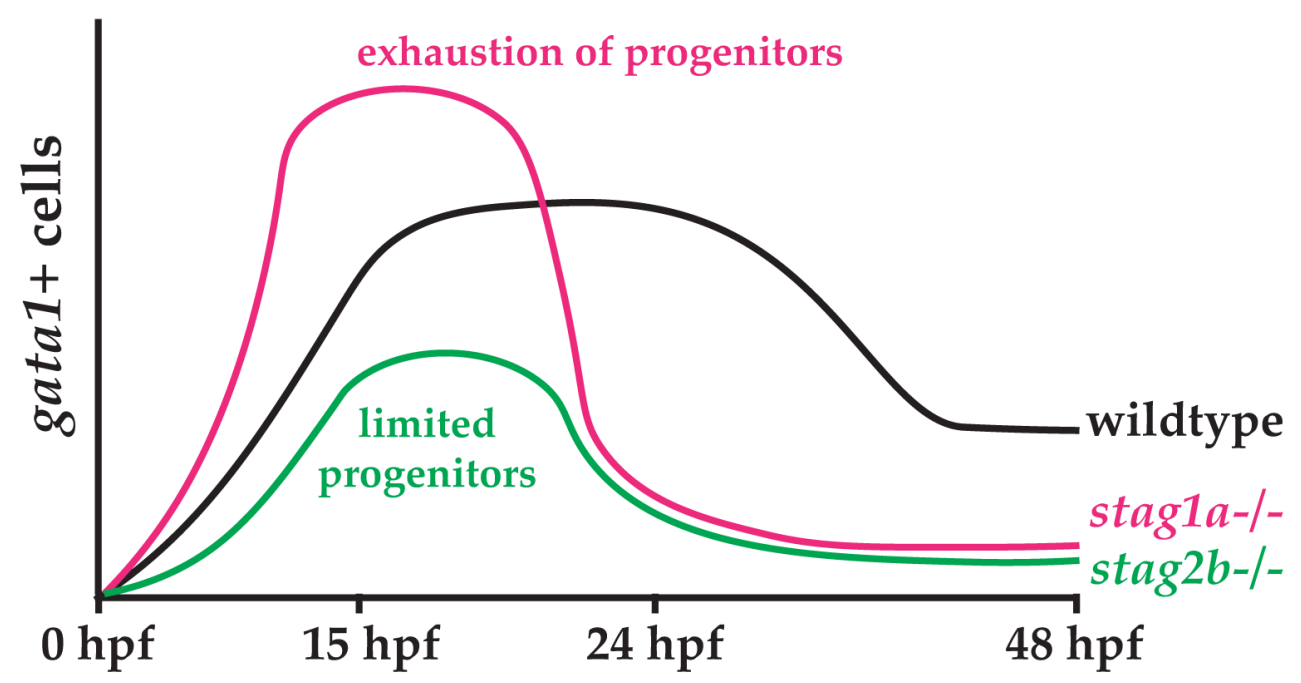

Figure 7. Hypothetical model explaining the effects of Stag1a and Stag2b loss on primitive erythropoiesis. In $\operatorname{stag} 1 a^{n z 204}$ mutants, an expansion of early haematopoietic progenitors driven by increased $s c l$ expression may lead to precocious differentiation that exhausts the progenitor pool. In stag $2 b^{n z 207}$ mutants, a limited pool of haematopoietic progenitors resulting from reduced $s c l$ expression leads to a reduction in primitive erythropoiesis.

A remaining question is the mechanism by which $\operatorname{stag} 1 a^{n z 204}$ and $\operatorname{stag} 2 b^{n z 207}$ mutants differentially affect $s c l$ expression. High levels of Bmp signalling induce lateral plate mesoderm and specify haematopoietic fate (Davidson and Zon, 2000; Prummel et al., 2020). Bmp signalling cooperates with Wnt signalling to promote blood fate through activation of homeobox transcription factors Cdx1 and $\mathrm{Cdx} 4$ (Lengerke et al., 2008). Previous studies show that mutations in cohesin subunits interfere with canonical Wnt signalling (Avagliano et al., 2017; Chin et al., 2020), so it is possible that loss of Stag1a or Stag2b differentially affect the balance of Bmp and Wnt signalling that directs the production of $s c l$-positive cells. Further experimentation will be needed to determine whether this is the case.

In summary, we have characterised the expression and function of zebrafish Stag paralogues in early development and haematopoiesis. We found a surprising role for the Stagla orthologue in restricting primitive vascular/haematopoietic cell numbers. In contrast, Stag2b loss-of-function reduced progenitor numbers. Subfunctionalisation and homozygous viability of the zebrafish stag mutants 
offer a unique opportunity to dissect cohesin's developmental functions in the absence of interference from cell cycle phenotypes.

\section{MATERIALS AND METHODS}

\section{Zebrafish maintenance}

354 Wild type (WIK) and mutant fish lines were maintained according to established protocols (Westerfield, 1995). Zebrafish procedures were carried out in accordance with the Otago Zebrafish Facility Standard Operating Procedures. Zebrafish mutant lines were developed under AUP-19-17 approved by the University of Otago Animal Ethics Committee. For all experiments, embryos were incubated at $22^{\circ} \mathrm{C}$ or $28^{\circ} \mathrm{C}$.

\section{CRISPR-Cas9 editing}

At least three sgRNAs were designed for each stag gene using the publicly available CHOPCHOP CRISPR design tool (Montague et al., 2014). sgRNAs were synthesised using a cloning-free approach as previously described (Varshney et al., 2016). Recombinant Cas9 protein was obtained commercially (PNA Bio Inc., Newbury Park, California, USA). Ribonucleoprotein complexes (RNPs) were assembled by mixing sgRNA and Cas9 protein at concentrations of $100 \mathrm{pg} / \mathrm{embryo}$ and $300 \mathrm{pg} / \mathrm{embryo}$, respectively in $300 \mathrm{mM} \mathrm{KCl}$. RNPs were incubated for 5 minutes at $37^{\circ} \mathrm{C}$ before injection into 1-cell stage WIK embryos. Editing efficiencies were evaluated by genotyping eight embryos from each injection clutch using high resolution melt analysis (HRMA). The most efficient sgRNAs were used to generate germline mutant lines (Supplementary Table 2). Primers used for genotyping are listed in Supplementary Table 3.

\section{Morpholino and mRNA rescue injections}

Morpholinos were purchased from Gene Tools LLC (Philomath, Oregon, USA) for the stag genes (Supplementary Table 4). 1-cell stage zebrafish embryos were injected with $0.5 \mathrm{mM}$ of morpholino. Full-length mRNA constructs in pcDNA3.1 $/ \mathrm{C}-(\mathrm{K}) \mathrm{DYK}$ vectors were obtained from GenScript Biotech (Piscataway, New Jersey, USA) for each stag gene. mRNA was synthesised using the mMessage mMachine transcription kit (Ambion, Austin, Texas, USA) and $200 \mathrm{pg}$ was injected into stag mutant embryos at the 1-cell stage.

\section{Whole-mount in situ hybridisation (WISH)}

379 WISH was performed as previously described (Thisse and Thisse, 2008). Digoxigenin-labelled 380 riboprobes for the four stag genes were synthesized from PCR clones inserted into pGEM®-T Easy vectors (Promega, Madison, Wisconsin, USA) using T7/Sp6 RNA polymerase (Roche Diagnostics, Basel, Switzerland). Anti-DIG alkaline phosphatase antibody (Roche Diagnostics, Basel, Switzerland) was used for detection, followed by visualization with nitro blue tetrazolium and 5bromo-4-chloro-3-indolylphosphate (NBT/BCIP) (Roche Diagnostics, Basel, Switzerland). Embryos were imaged using a Leica M205 FA epifluorescence microscope (Leica, Wetzlar, Germany Applications Suite). Primers used for the amplification of stag riboprobes are listed in Supplementary Table 3. 


\section{Quantitative PCR (qPCR)}

389 Total mRNA was extracted from pools of 30 embryos using NucleoSpin RNA kit (Macherey-Nagel,

390 Bethlehem, PA, USA). Complementary DNA (cDNA) was synthesized with qScript cDNA

391 SuperMix (Quanta Biosciences, Beverly, MA, USA). Expression levels of the stag paralogues

392 (primer sequences in Supplementary Table S3) and haematopoietic markers were measured using

393 SYBR Premix Ex Taq II (Takara Bio Inc., Kusatsu, Japan) on a Roche LightCycler400. Reference

394 genes were $b$-actin and rpll3a.

\section{Hybridisation chain reaction (HCR)}

396 HCR probe sets for pax2, scl, runx1, gata1, spil and fli1 were obtained from Molecular Instruments,

397 Inc. (California, USA). HCR was performed as per the manufacturer's protocol for zebrafish

398 embryos. Embryos were mounted in 1\% agarose and imaged on Nikon C2 confocal microscope

399 (Nikon Corp, Tokyo, Japan NIS-Elements). Image analysis was performed using ImageJ. For

400 embryos shown in figures, maximum intensity projections were generated and brightness/contrast

401

402

403

404 was adjusted with no further processing. For quantitative analysis, individual channels were background-subtracted, auto-thresholded using the RenyiEntropy algorithm (Kapur et al., 1985) and fluorescence intensities were measured. Colocalization analysis was performed using the JACoP plugin (Bolte and Cordelières, 2006) in ImageJ.

\section{Statistical Analysis}

GraphPad PRISM 7 was used for performing all statistical analysis. One-way ANOVAs (Tukey's multiple comparisons tests) were used for estimating the statistical significance of qPCR and HCR data.

\section{CONFLICTS OF INTEREST}

411 The authors declare that the research was conducted in the absence of any commercial or financial relationships that could be construed as a potential conflict of interest.

\section{AUTHOR CONTRIBUTIONS}

415 SK and JAH designed experiments. SK and AL performed experiments. SK, AL and JAH analyzed data. SK and JAH wrote the paper. All authors read and approved the final manuscript.

419 This research was funded by a Royal Society of NZ Marsden Fund Grant 16-UOO-072, a Leukaemia 420 and Blood Cancer NZ National Research Grant, and a Maurice Wilkins Centre for Molecular

421 Biodiscovery Research Grant. 


\section{ACKNOWLEDGEMENTS}

424

The authors would like to thank Noel Jhinku for expert management of the zebrafish facility, and Jisha Antony for helpful advice and discussions.

\section{REFERENCES}

Antony, J., Gimenez, G., Taylor, T., Khatoon, U., Day, R., Morison, I.M., et al. (2020). BET inhibition prevents aberrant RUNX1 and ERG transcription in STAG2 mutant leukaemia cells. J Mol Cell Biol 12(5), 397-399. doi: 10.1093/jmcb/mjz114.

Avagliano, L., Grazioli, P., Mariani, M., Bulfamante, G.P., Selicorni, A., and Massa, V. (2017). Integrating molecular and structural findings: Wnt as a possible actor in shaping cognitive impairment in Cornelia de Lange syndrome. Orphanet J Rare Dis 12(1), 174. doi: 10.1186/s13023-017-0723-0.

Berman, J.N., Kanki, J.P., and Look, A.T. (2005). Zebrafish as a model for myelopoiesis during embryogenesis. Exp Hematol 33(9), 997-1006.

Bolte, S., and Cordelières, F.P. (2006). A guided tour into subcellular colocalization analysis in light microscopy. J Microsc 224(Pt 3), 213-232. doi: 10.1111/j.1365-2818.2006.01706.x.

Bonora, G., Plath, K., and Denholtz, M. (2014). A mechanistic link between gene regulation and genome architecture in mammalian development. Curr Opin Genet Dev 27, 92-101. doi: 10.1016/j.gde.2014.05.002.

Chin, C.V., Antony, J., Ketharnathan, S., Gimenez, G., Parsons, K.M., He, J., et al. (2020). Cohesin mutations are synthetic lethal with stimulation of WNT signaling. bioRxiv, 2020.2007.2023.218875. doi: 10.1101/2020.07.23.218875.

Cuadrado, A., and Losada, A. (2020). Specialized functions of cohesins STAG1 and STAG2 in 3D genome architecture. Curr Opin Genet Dev 61, 9-16. doi: 10.1016/j.gde.2020.02.024.

Davidson, A.J., and Zon, L.I. (2000). Turning mesoderm into blood: the formation of hematopoietic stem cells during embryogenesis [In Process Citation]. Curr Top Dev Biol 50, 45-60.

De Koninck, M., Lapi, E., Badía-Careaga, C., Cossío, I., Giménez-Llorente, D., Rodríguez-Corsino, M., et al. (2020). Essential Roles of Cohesin STAG2 in Mouse Embryonic Development and Adult Tissue Homeostasis. Cell Reports 32(6), 108014. doi: https://doi.org/10.1016/j.celrep.2020.108014.

De Koninck, M., and Losada, A. (2016). Cohesin Mutations in Cancer. Cold Spring Harb Perspect Med 6(12). doi: 10.1101/cshperspect.a026476.

Dorsett, D. (2009). Cohesin, gene expression and development: lessons from Drosophila. Chromosome Res 17(2), 185-200.

Dorsett, D., Eissenberg, J.C., Misulovin, Z., Martens, A., Redding, B., and McKim, K. (2005). Effects of sister chromatid cohesion proteins on cut gene expression during wing development in Drosophila. Development 132(21), 4743-4753. doi: 10.1242/dev.02064.

Dorsett, D., and Strom, L. (2012). The ancient and evolving roles of cohesin in gene expression and DNA repair. Curr Biol 22(7), R240-250. doi: 10.1016/j.cub.2012.02.046. 
462

463

464

465

466

467

468

469

470

471

472

473

474

475

476

477

478

479

480

481

482

483

484

485

486

487

488

489

490

491

492

493

494

495

496

497

498

499

500

501

Downing, J.R., Higuchi, M., Lenny, N., and Yeoh, A.E. (2000). Alterations of the AML1 transcription factor in human leukemia. Semin Cell Dev Biol 11(5), 347-360.

Fudenberg, G., Abdennur, N., Imakaev, M., Goloborodko, A., and Mirny, L.A. (2018). Emerging Evidence of Chromosome Folding by Loop Extrusion. Cold Spring Harb Symp Quant Biol. doi: $10.1101 /$ sqb.2017.82.034710.

Galeev, R., Baudet, A., Kumar, P., Rundberg Nilsson, A., Nilsson, B., Soneji, S., et al. (2016). Genome-wide RNAi Screen Identifies Cohesin Genes as Modifiers of Renewal and Differentiation in Human HSCs. Cell Reports 14(12), 2988-3000. doi: 10.1016/j.celrep.2016.02.082.

Gause, M., Misulovin, Z., Bilyeu, A., and Dorsett, D. (2010). Dosage-sensitive regulation of cohesin chromosome binding and dynamics by Nipped-B, Pds5, and Wapl. Mol Cell Biol 30(20), 4940-4951. doi: MCB.00642-10 [pii]

\subsection{8/MCB.00642-10.}

Gering, M., Rodaway, A.R., Gottgens, B., Patient, R.K., and Green, A.R. (1998). The SCL gene specifies haemangioblast development from early mesoderm. EMBOJ 17(14), 4029-4045. doi: 10.1093/emboj/17.14.4029.

Hansen, A.S. (2020). CTCF as a boundary factor for cohesin-mediated loop extrusion: evidence for a multi-step mechanism. Nucleus 11(1), 132-148. doi: 10.1080/19491034.2020.1782024.

Hill, V.K., Kim, J.S., and Waldman, T. (2016). Cohesin mutations in human cancer. Biochim Biophys Acta 1866(1), 1-11. doi: 10.1016/j.bbcan.2016.05.002.

Hnisz, D., Day, D.S., and Young, R.A. (2016). Insulated Neighborhoods: Structural and Functional Units of Mammalian Gene Control. Cell 167(5), 1188-1200. doi: http://dx.doi.org/10.1016/j.cell.2016.10.024.

Horsfield, J.A., Anagnostou, S.H., Hu, J.K., Cho, K.H., Geisler, R., Lieschke, G., et al. (2007). Cohesin-dependent regulation of Runx genes. Development 134(14), 2639-2649. doi: 10.1242/dev.002485.

Horsfield, J.A., Print, C.G., and Monnich, M. (2012). Diverse developmental disorders from the one ring: distinct molecular pathways underlie the cohesinopathies. Front Genet 3, 171. doi: 10.3389/fgene.2012.00171.

Kapur, J.N., Sahoo, P.K., and Wong, A.K.C. (1985). A new method for gray-level picture thresholding using the entropy of the histogram. Computer Vision, Graphics, and Image Processing 29(3), 273-285. doi: https://doi.org/10.1016/0734-189X(85)90125-2.

Kawauchi, S., Calof, A.L., Santos, R., Lopez-Burks, M.E., Young, C.M., Hoang, M.P., et al. (2009). Multiple organ system defects and transcriptional dysregulation in the Nipbl(+/-) mouse, a model of Cornelia de Lange Syndrome. PLoS Genet 5(9), e1000650. doi: 10.1371/journal.pgen.1000650.

Kawauchi, S., Santos, R., Muto, A., Lopez-Burks, M.E., Schilling, T.F., Lander, A.D., et al. (2016). Using mouse and zebrafish models to understand the etiology of developmental defects in Cornelia de Lange Syndrome. Am J Med Genet C Semin Med Genet 172(2), 138-145. doi: 10.1002/ajmg.c.31484. 
Kon, A., Shih, L.Y., Minamino, M., Sanada, M., Shiraishi, Y., Nagata, Y., et al. (2013). Recurrent mutations in multiple components of the cohesin complex in myeloid neoplasms. Nat Genet 45. doi: 10.1038/ng.2731.

Leeke, B., Marsman, J., O'Sullivan, J.M., and Horsfield, J.A. (2014). Cohesin mutations in myeloid malignancies: underlying mechanisms. Exp Hematol Oncol 3, 13. doi: 10.1186/2162-3619-313.

Lengerke, C., Schmitt, S., Bowman, T.V., Jang, I.H., Maouche-Chretien, L., McKinney-Freeman, S., et al. (2008). BMP and Wnt specify hematopoietic fate by activation of the Cdx-Hox pathway. Cell Stem Cell 2(1), 72-82. doi: 10.1016/j.stem.2007.10.022.

Liu, J., and Krantz, I.D. (2009). Cornelia de Lange syndrome, cohesin, and beyond. Clinical genetics 76(4), 303-314. doi: 10.1111/j.1399-0004.2009.01271.x.

Liu, Y., Xu, H., Van der Jeught, K., Li, Y., Liu, S., Zhang, L., et al. (2018). Somatic mutation of the cohesin complex subunit confers therapeutic vulnerabilities in cancer. J Clin Invest 128(7), 2951-2965. doi: 10.1172/JCI98727.

Losada, A. (2008). The regulation of sister chromatid cohesion. Biochim Biophys Acta 1786(1), 4148. doi: 10.1016/j.bbcan.2008.04.003.

Marsman, J., O'Neill, A.C., Kao, B.R., Rhodes, J.M., Meier, M., Antony, J., et al. (2014). Cohesin and CTCF differentially regulate spatiotemporal runx1 expression during zebrafish development. Biochim Biophys Acta 1839(1), 50-61. doi: 10.1016/j.bbagrm.2013.11.007.

Mazumdar, C., Shen, Y., Xavy, S., Zhao, F., Reinisch, A., Li, R., et al. (2015). Leukemia-Associated Cohesin Mutants Dominantly Enforce Stem Cell Programs and Impair Human Hematopoietic Progenitor Differentiation. Cell Stem Cell 17(6), 675-688. doi: 10.1016/j.stem.2015.09.017.

Merkenschlager, M. (2010). Cohesin: a global player in chromosome biology with local ties to gene regulation. Curr Opin Genet Dev 20(5), 555-561. doi: S0959-437X(10)00096-1 [pii]

10.1016/j.gde.2010.05.007.

Merkenschlager, M., and Odom, D.T. (2013). CTCF and cohesin: linking gene regulatory elements with their targets. Cell 152(6), 1285-1297. doi: 10.1016/j.cell.2013.02.029.

Monnich, M., Banks, S., Eccles, M., Dickinson, E., and Horsfield, J. (2009). Expression of cohesin and condensin genes during zebrafish development supports a non-proliferative role for cohesin. Gene Expr Patterns 9(8), 586-594. doi: 10.1016/j.gep.2009.08.004.

Montague, T.G., Cruz, J.M., Gagnon, J.A., Church, G.M., and Valen, E. (2014). CHOPCHOP: a CRISPR/Cas9 and TALEN web tool for genome editing. Nucleic Acids Res 42(Web Server issue), W401-407. doi: 10.1093/nar/gku410.

Mullenders, J., Aranda-Orgilles, B., Lhoumaud, P., Keller, M., Pae, J., Wang, K., et al. (2015). Cohesin loss alters adult hematopoietic stem cell homeostasis, leading to myeloproliferative neoplasms. J Exp Med 212(11), 1833-1850. doi: 10.1084/jem.20151323.

Muto, A., Calof, A.L., Lander, A.D., and Schilling, T.F. (2011). Multifactorial origins of heart and gut defects in nipbl-deficient zebrafish, a model of Cornelia de Lange Syndrome. PLoS biology 9(10), e1001181. doi: 10.1371/journal.pbio.1001181.

Muto, A., Ikeda, S., Lopez-Burks, M.E., Kikuchi, Y., Calof, A.L., Lander, A.D., et al. (2014). Nipb1 and mediator cooperatively regulate gene expression to control limb development. PLoS Genet 10(9), e1004671. doi: 10.1371/journal.pgen.1004671. 
544

545

546

547

548

549

550

551

552

553

554

555

556

557

558

559

560

561

562

563

564

565

566

567

568

569

570

571

572

573

574

575

576

577

578

579

580

581

582

583

584

585

Nasmyth, K. (2011). Cohesin: a catenase with separate entry and exit gates? Nature cell biology 13(10), 1170-1177. doi: 10.1038/ncb2349.

Nasmyth, K., and Haering, C.H. (2009). Cohesin: its roles and mechanisms. Annu Rev Genet 43, 525558. doi: 10.1146/annurev-genet-102108-134233.

Newkirk, D.A., Chen, Y.Y., Chien, R., Zeng, W., Biesinger, J., Flowers, E., et al. (2017). The effect of Nipped-B-like (Nipbl) haploinsufficiency on genome-wide cohesin binding and target gene expression: modeling Cornelia de Lange syndrome. Clin Epigenetics 9, 89. doi: 10.1186/s13148-017-0391-x.

Onn, I., Heidinger-Pauli, J.M., Guacci, V., Unal, E., and Koshland, D.E. (2008). Sister chromatid cohesion: a simple concept with a complex reality. Annu Rev Cell Dev Biol 24, 105-129. doi: 10.1146/annurev.cellbio.24.110707.175350.

Orkin, S.H. (1995). Hematopoiesis: how does it happen? Curr Opin Cell Biol 7(6), 870-877. doi: 10.1016/0955-0674(95)80072-7.

Papaemmanuil, E., Gerstung, M., Bullinger, L., Gaidzik, V.I., Paschka, P., Roberts, N.D., et al. (2016). Genomic Classification and Prognosis in Acute Myeloid Leukemia. New England Journal of Medicine 374(23), 2209-2221. doi: doi:10.1056/NEJMoa1516192.

Prummel, K.D., Nieuwenhuize, S., and Mosimann, C. (2020). The lateral plate mesoderm. Development 147(12), dev175059. doi: 10.1242/dev.175059.

Remeseiro, S., Cuadrado, A., Carretero, M., Martinez, P., Drosopoulos, W.C., Canamero, M., et al. (2012a). Cohesin-SA1 deficiency drives aneuploidy and tumourigenesis in mice due to impaired replication of telomeres. The EMBO journal 31(9), 2076-2089. doi: 10.1038/emboj.2012.11.

Remeseiro, S., Cuadrado, A., Gomez-Lopez, G., Pisano, D.G., and Losada, A. (2012b). A unique role of cohesin-SA1 in gene regulation and development. EMBO J 31(9), 2090-2102. doi: 10.1038/emboj.2012.60.

Rhodes, J.M., Bentley, F.K., Print, C.G., Dorsett, D., Misulovin, Z., Dickinson, E.J., et al. (2010). Positive regulation of c-Myc by cohesin is direct, and evolutionarily conserved. Dev Biol 344(2), 637-649. doi: 10.1016/j.ydbio.2010.05.493.

Rowley, M.J., and Corces, V.G. (2018). Organizational principles of 3D genome architecture. Nat Rev Genet 19(12), 789-800. doi: 10.1038/s41576-018-0060-8.

Shi, Z., Gao, H., Bai, X.C., and Yu, H. (2020). Cryo-EM structure of the human cohesin-NIPBLDNA complex. Science 368(6498), 1454-1459. doi: 10.1126/science.abb0981.

Smith, T.G., Laval, S., Chen, F., Rock, M.J., Strachan, T., and Peters, H. (2014). Neural crest cellspecific inactivation of Nipbl or Mau2 during mouse development results in a late onset of craniofacial defects. Genesis 52(7), 687-694. doi: 10.1002/dvg.22780.

Speck, N.A. (2001). Core binding factor and its role in normal hematopoietic development. Curr Opin Hematol 8(4), 192-196.

Thisse, C., and Thisse, B. (2008). High-resolution in situ hybridization to whole-mount zebrafish embryos. Nat Protoc 3(1), 59-69. doi: 10.1038/nprot.2007.514.

Thol, F., Bollin, R., Gehlhaar, M., Walter, C., Dugas, M., Suchanek, K.J., et al. (2014). Mutations in the cohesin complex in acute myeloid leukemia: clinical and prognostic implications. Blood 123. doi: 10.1182/blood-2013-07-518746. 
586

587

588

589

590

591

592

593

594

595

596

597

598

599

600

601

602

603

604

605

606

607

608

609

610

611

612

613
Thota, S., Viny, A.D., Makishima, H., Spitzer, B., Radivoyevitch, T., Przychodzen, B., et al. (2014). Genetic alterations of the cohesin complex genes in myeloid malignancies. Blood 124(11), 1790-1798. doi: 10.1182/blood-2014-04-567057.

van der Lelij, P., Lieb, S., Jude, J., Wutz, G., Santos, C.P., Falkenberg, K., et al. (2017). Synthetic lethality between the cohesin subunits STAG1 and STAG2 in diverse cancer contexts. Elife 6. doi: $10.7554 /$ eLife.26980.

Varshney, G.K., Carrington, B., Pei, W., Bishop, K., Chen, Z., Fan, C., et al. (2016). A highthroughput functional genomics workflow based on CRISPR/Cas9-mediated targeted mutagenesis in zebrafish. Nat Protoc 11(12), 2357-2375. doi: 10.1038/nprot.2016.141.

Vietri Rudan, M., and Hadjur, S. (2015). Genetic Tailors: CTCF and Cohesin Shape the Genome During Evolution. Trends Genet 31(11), 651-660. doi: 10.1016/j.tig.2015.09.004.

Viny, A.D., Bowman, R.L., Liu, Y., Lavallee, V.P., Eisman, S.E., Xiao, W., et al. (2019). Cohesin Members Stag1 and Stag2 Display Distinct Roles in Chromatin Accessibility and Topological Control of HSC Self-Renewal and Differentiation. Cell Stem Cell 25(5), 682-696 e688. doi: 10.1016/j.stem.2019.08.003.

Viny, A.D., and Levine, R.L. (2018). Cohesin mutations in myeloid malignancies made simple. Curr Opin Hematol 25(2), 61-66. doi: 10.1097/MOH.0000000000000405.

Viny, A.D., Ott, C.J., Spitzer, B., Rivas, M., Meydan, C., Papalexi, E., et al. (2015). Dose-dependent role of the cohesin complex in normal and malignant hematopoiesis. $J$ Exp Med 212(11), 1819-1832. doi: 10.1084/jem.20151317.

Waldman, T. (2020). Emerging themes in cohesin cancer biology. Nat Rev Cancer. doi: 10.1038/s41568-020-0270-1.

Westerfield, M. (1995). The Zebrafish Book. A guide for the laboratory use of zebrafish (Brachydanio rerio). Eugene, Oregon: University of Oregon Press.

Yoshida, K., Toki, T., Okuno, Y., Kanezaki, R., Shiraishi, Y., Sato-Otsubo, A., et al. (2013). The landscape of somatic mutations in Down syndrome-related myeloid disorders. Nature genetics. doi: 10.1038/ng.2759. 
biof6i preprint doi: https://doi.org/10.1101/2020.10.19.346122; this version postę October 19, 2020. The copyright holder for this preprint (which uas not certified by peer review) is the author/funder, who has granted bioRx v a license to display the preprint in perpetuity. It is made
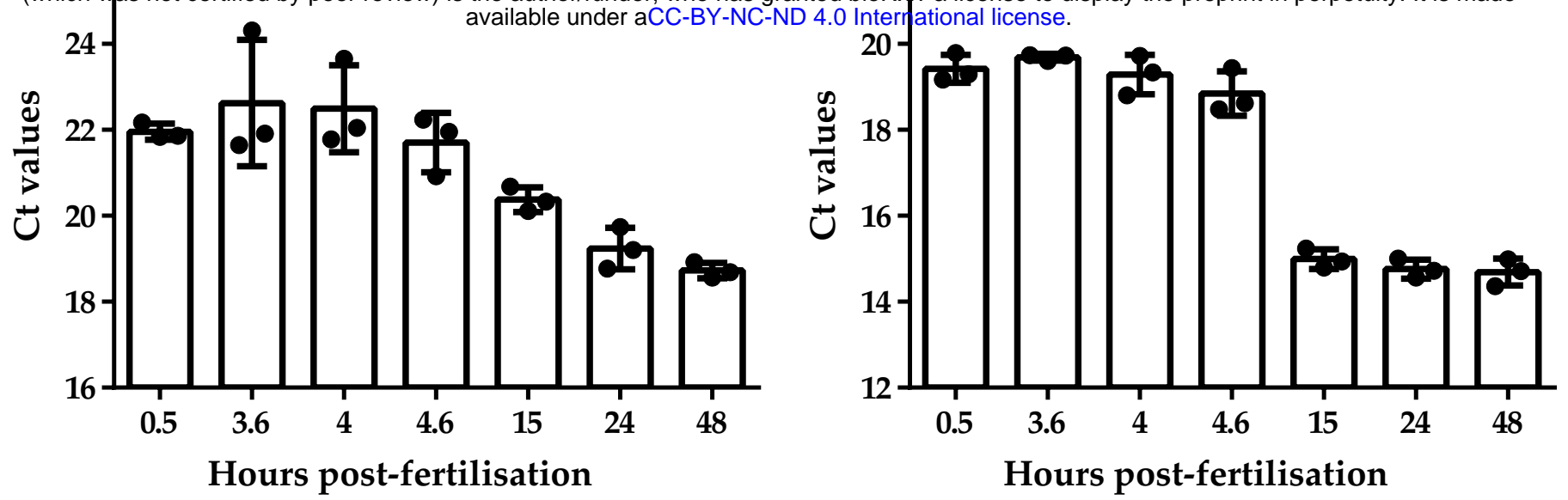

B
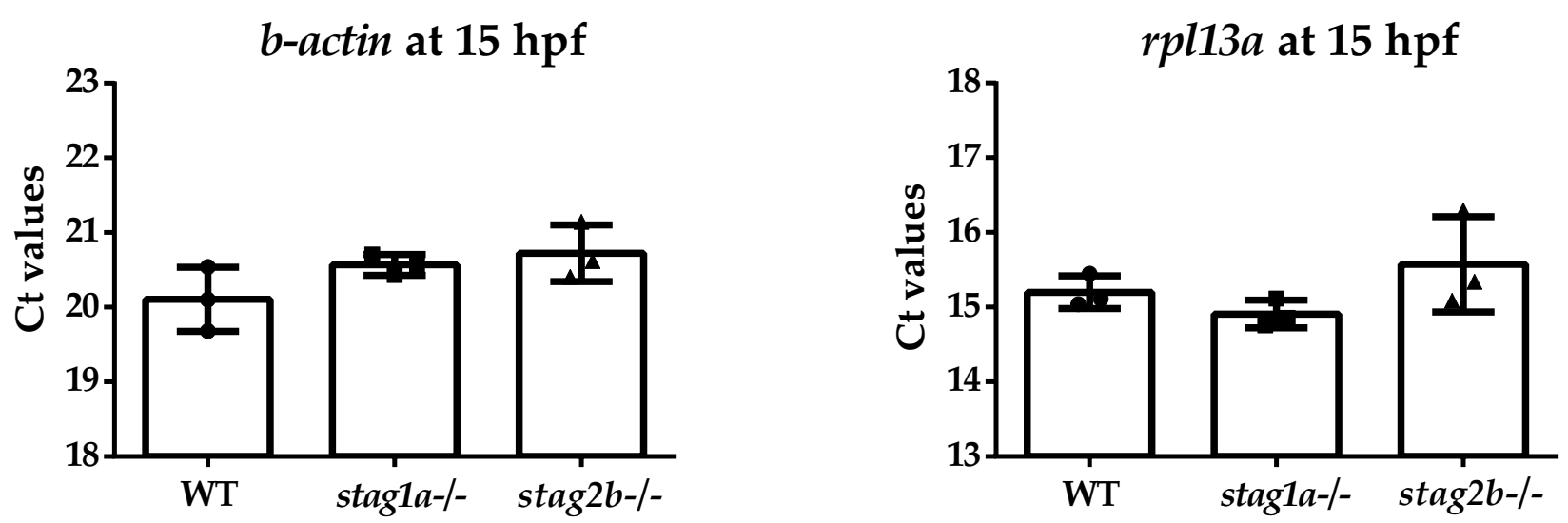

C

b-actin at $48 \mathrm{hpf}$
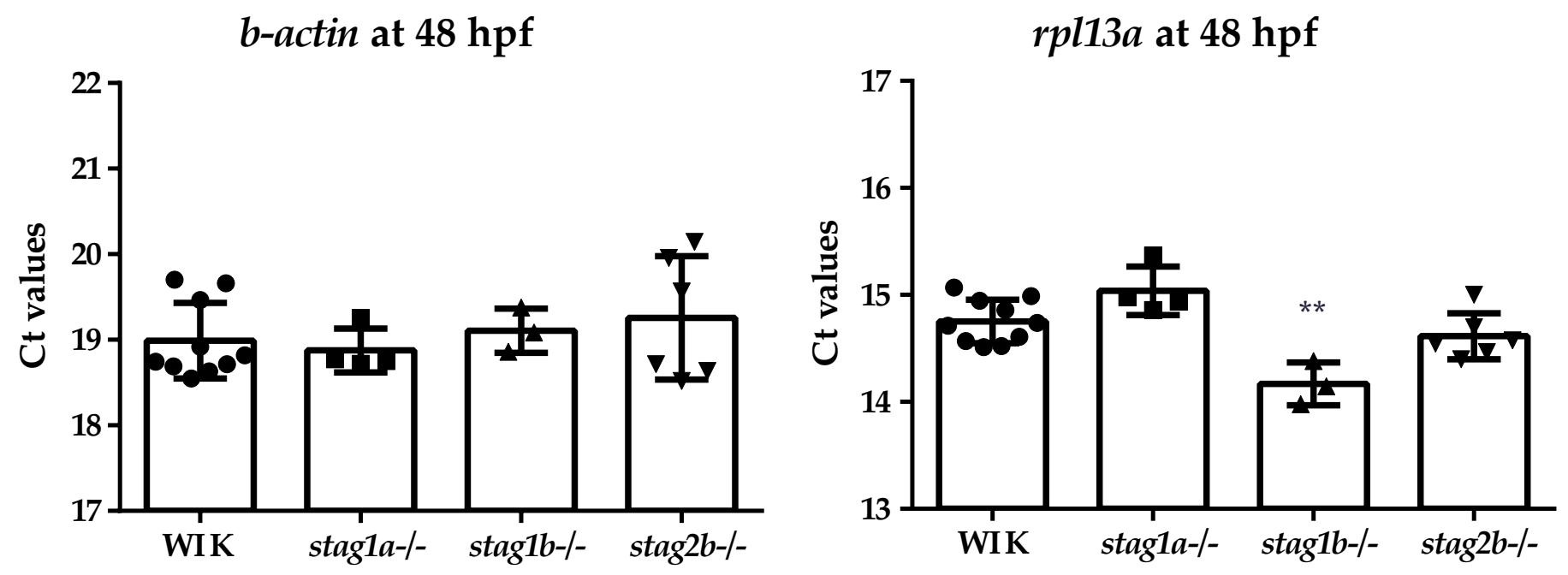

Supplementary Figure 1. Stability of reference genes used for quantitative RT-PCR (qPCR) normalisation. (A) Ct values of $b$-actin and rpl13a in wildtype embryos at $48 \mathrm{hpf}$. (B) Ct values of b-actin and rpl13a in wildtype and mutant embryos at $15 \mathrm{hpf}$ (C) Ct values of b-actin and rpl13a in wildtype and mutant embryos at $48 \mathrm{hpf}$. Expression of $r p l 13 a$ is altered in mutants. ${ }^{* *} \mathrm{P} \leq 0.01$; one-way ANOVA. 
bidRxiv preprint doi: https://doi.org/10.1101/2020;010.19.346122; this version posted Octoberod 9, 2020. The copyright holder for this preprint

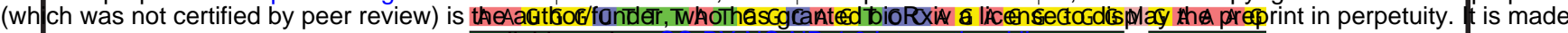

stag1a+l+
stag1a-1-
reference

$13 \mathrm{bp}$ deletion

GRCz10/danRer10 Chr 24

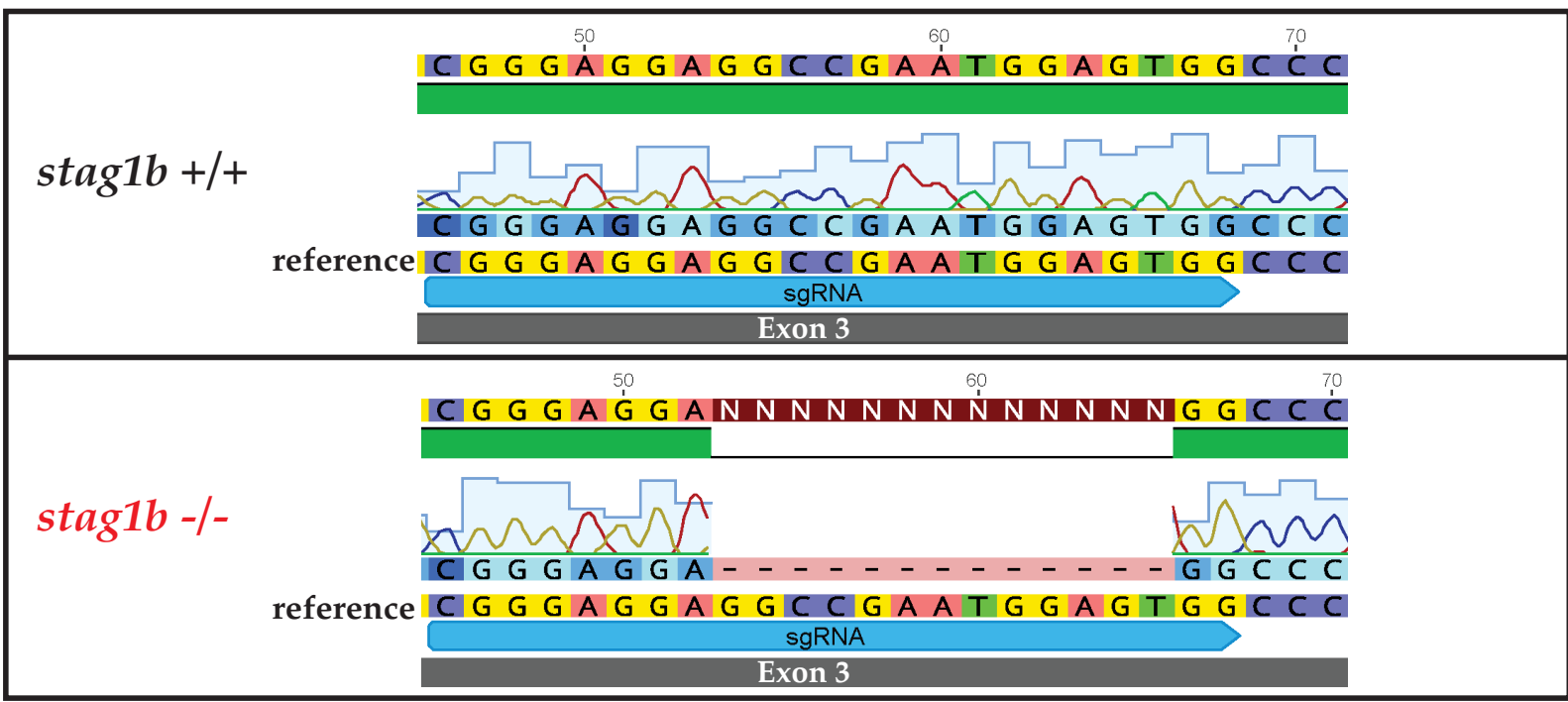

$7 \mathrm{bp}$ deletion

GRCz10/danRer10

Chr 14

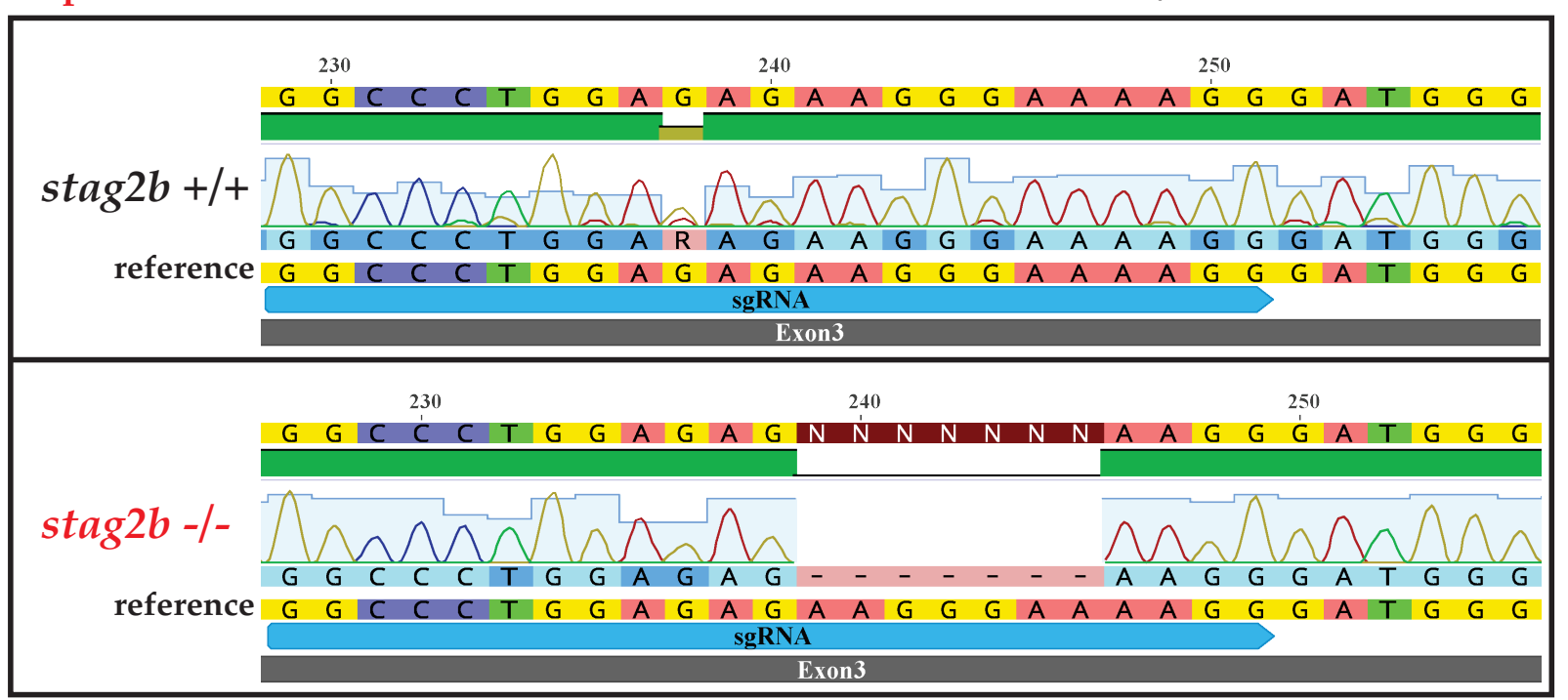

Supplementary Figure 2. Genomic DNA sequence detail of zebrafish stag gene germline CRISPR mutants. Nucleotide alignments of wildtype and mutant homozygous sequences are shown. The sgRNA sites are annotated in blue. 
bioRxiv preprint doi: https://doi.org/10.1101/2020.10.19.346122; this version posted October 19, 2020. The copyright holder for this preprint (which was not certified by peer review) is the author/funder, who has granted bioRxiv a license to display the preprint in perpetuity. It is made available under aCC-BY-NC-ND 4.0 International license.

A

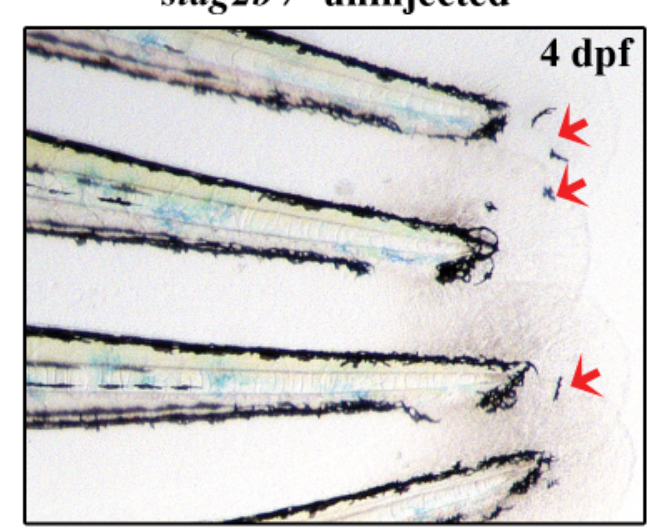

Ectopic pigments, $n=70 / 85$ stag $2 b-/-$ +200 pg stag $2 b$ mRNA

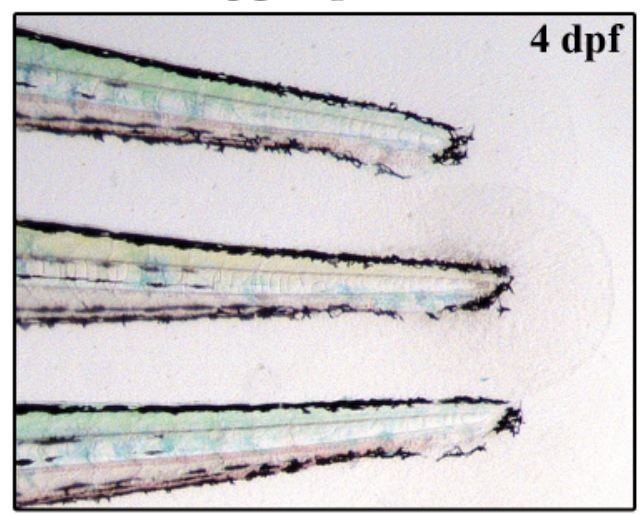

Normal, $n=35 / 61$

\section{B stag $2 b-/-$ injected with $0.5 \mathrm{mM}$ stag2b morpholino}
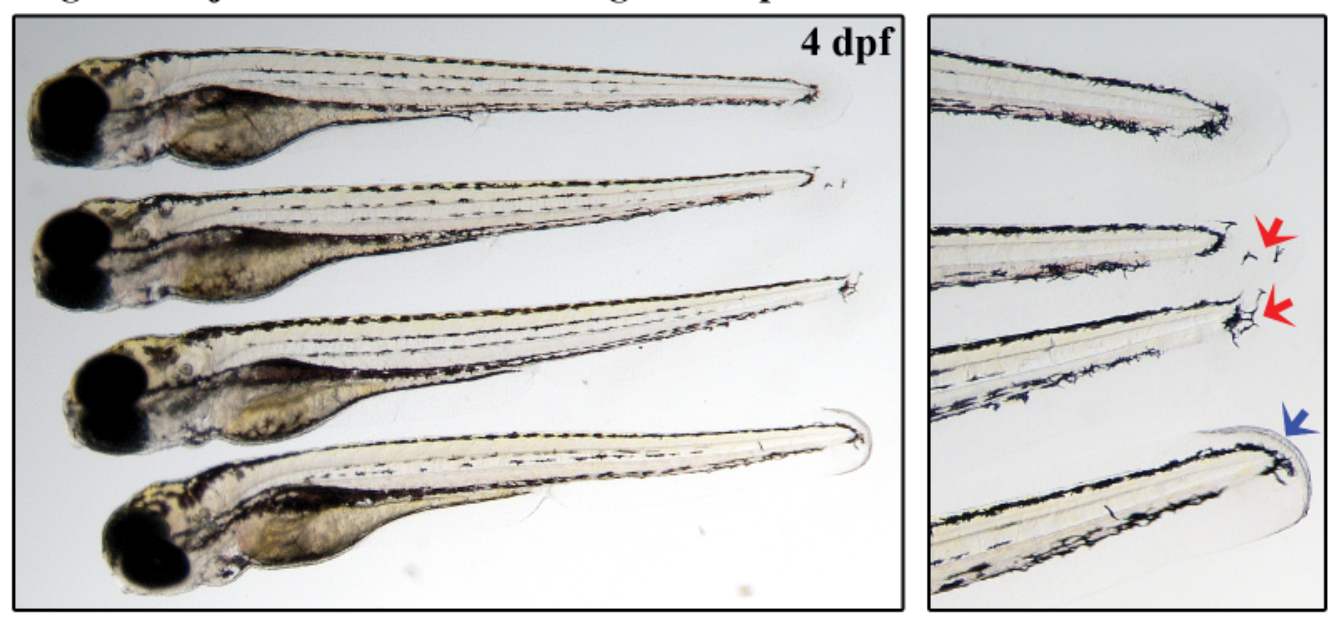

Ectopic pigments, $n=89 / 103$

Supplementary Figure 3. The stag2b mutation: confirmation of loss of function and and mutant phenotype. (A) The ectopic pigment cells seen in the tail fin of $s t a g 2 b$-/- embryos (red arrows) are rescued upon injection of stag $2 b$ mRNA. Lateral views of tail fin zoom-ins are shown, anterior to the left. (B) Injection of $0.5 \mathrm{mM}$ stag $2 b$ morpholino does not induce any additional phenotypes in stag $2 b-/-$ embryos, and confirms that Stag2 loss causes displaced pigment cells (red arrows) and tail fin folds (blue arrow). Lateral views of full-length embryos and tail fin zoom-ins are shown, anterior to the left. Numbers of embryos are indicated below the respective panels. 
bioRxiv preprint doi: https://doi.org/10.1101/2020.10.19.346122; this version posted October 19, 2020. The copyright holder for this preprint (which was not certified by peer review) is the author/funder, who has granted bioRxiv a license to display the preprint in perpetuity. It is made available under aCC-BY-NC-ND 4.0 International license.
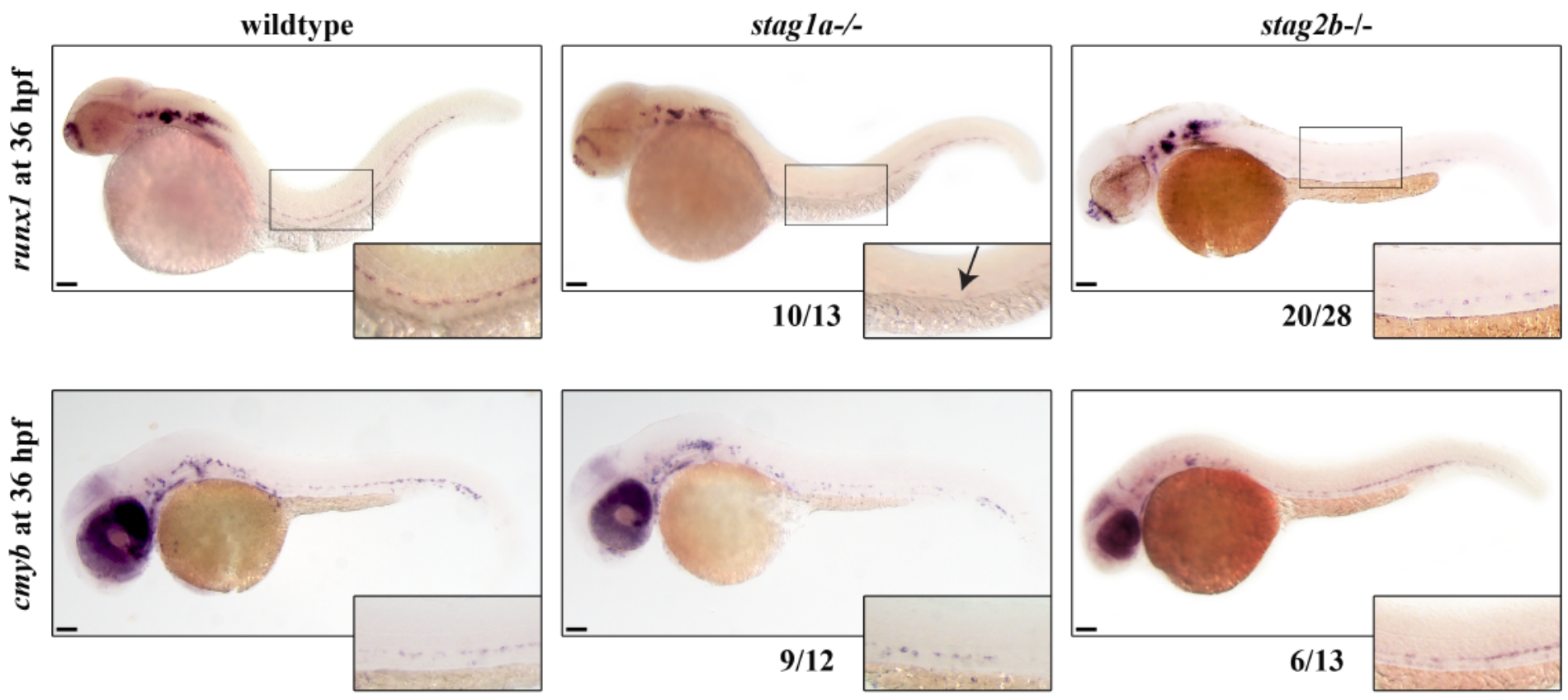

Supplementary Figure 4. Whole-mount in situ hybridization analysis of expression of runx 1 and $c m y b$ at $36 \mathrm{hpf}$. Lateral views are shown, anterior to the left. Insets show zoom-ins of the dorsal aorta region. Reduced runx1 expression in stag1a-/- embryos is indicated by an arrow. Number of embryos is indicated below the respective panels. Scale bars are $100 \mu \mathrm{m}$. 


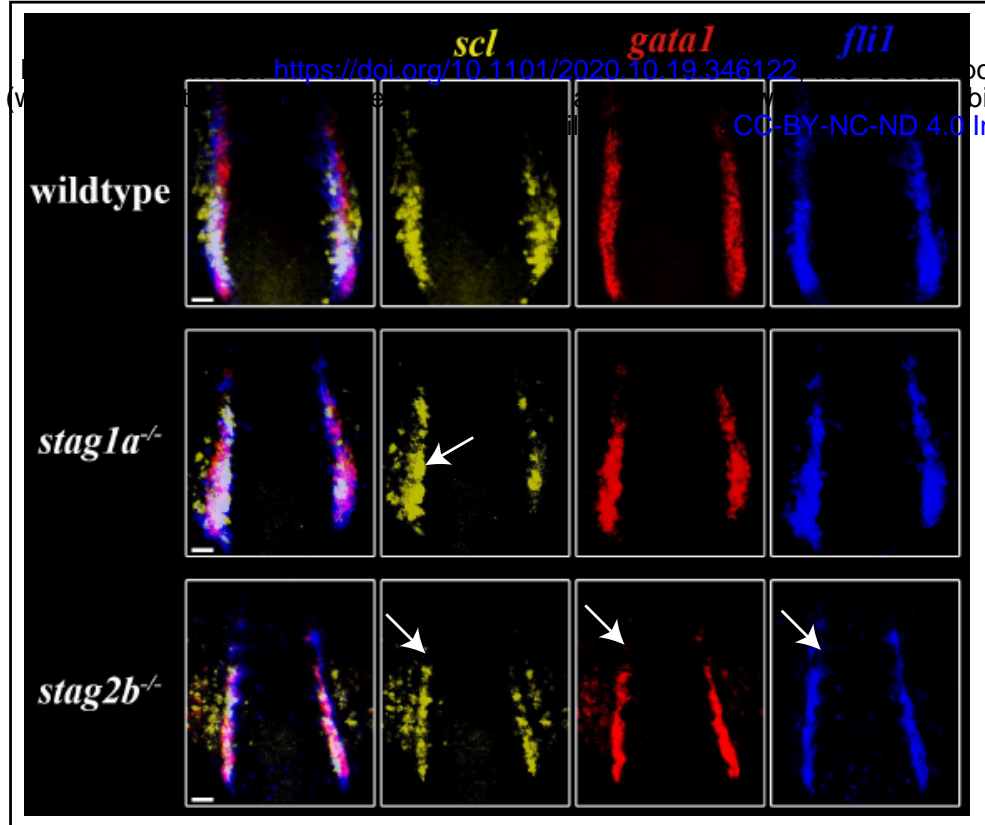

PLM composition $s c l$ expression in PLM osted Qobobov 19, 2020. The copyright holder for this preprint pion a license to display the preprint in perpetuity. It is made
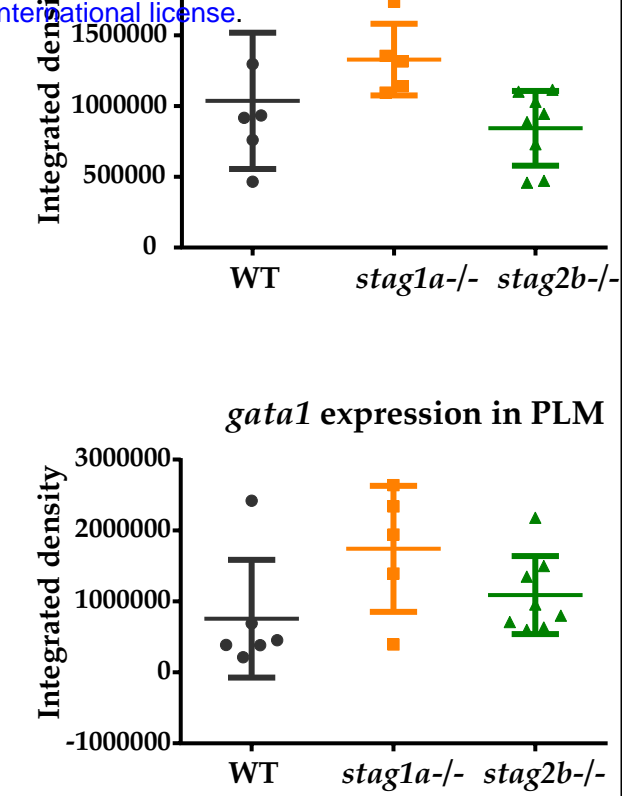

fli1 expression in PLM

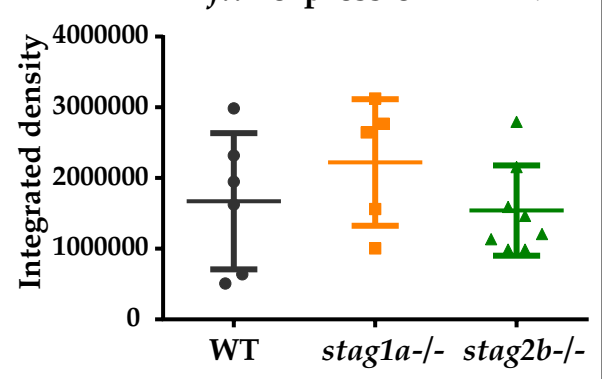

B

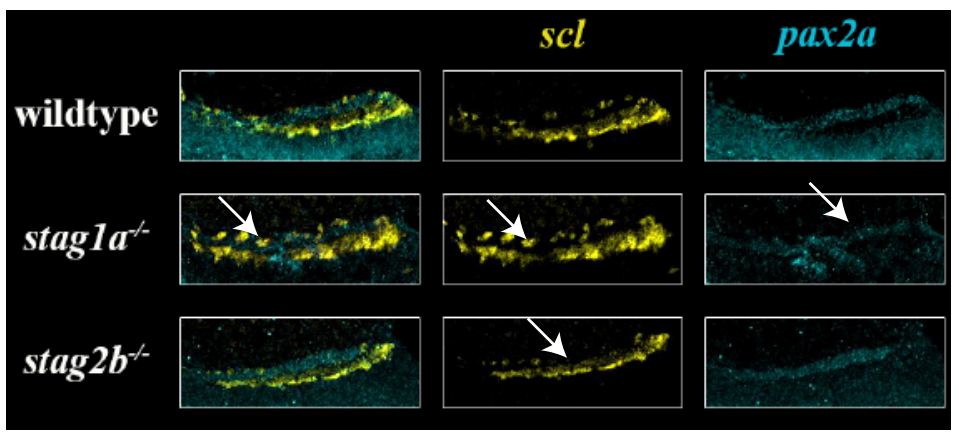

Supplementary Figure 5. Stag mutations affect gene expression in the posterior lateral mesoderm (PLM) and anterior lateral mesoderm (ALM). (A) Multiplexed in situ HCR of scl (yellow), gata1 (red), and fli1 (blue) expression at $15 \mathrm{hpf}$. Dorsal PLM views are shown, anterior to the top. Extra $s c l$ expression in stag1a-/- embryos and reduction of PLM expression in stag $2 b$-/- embryos is marked by white arrows. Quantitative analysis of fluor-
C

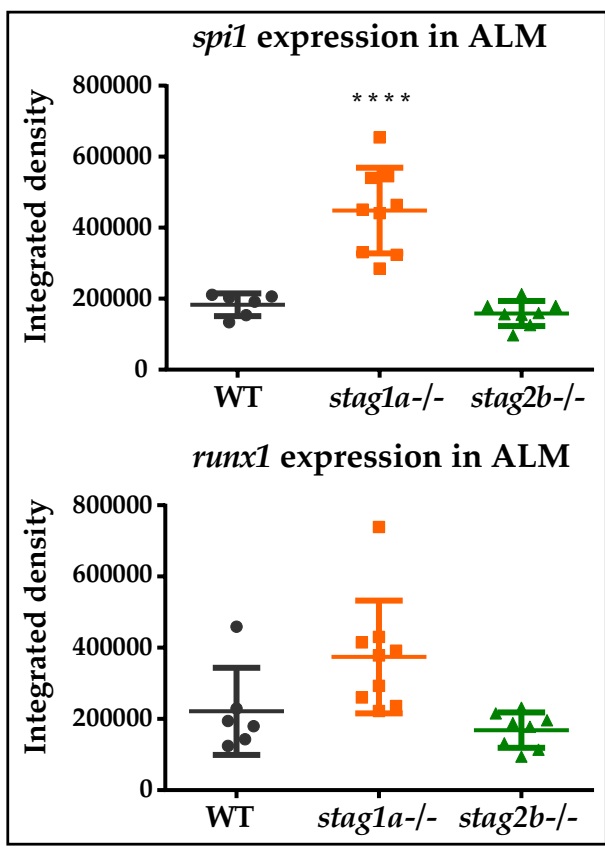

escence integrated densities indicates $s c l$, gata1 and fli1 trend to non-significant upregulation in stag1a-/- embryos in the PLM. Composition of the PLM is equivalent in all embryos. (B) Multiplexed in situ HCR of scl (yellow) and pax2a (cyan) expression at $15 \mathrm{hpf}$. Posterior views of a single PLM stripe are shown, dorsal to the left. Arrows mark lateral expansion of $s c l$ into the pax $2 a$ domain in the middle stag1a-/- panels and reduced scl expression in the lower stag2b-/- panel. (C) Quantitative analysis of fluorescence integrated densities of ALM markers shows an increase of runx1 and spil expression in stag1a-/- embryos. ${ }^{* * *} \mathrm{P} \leq 0.0001$; one-way ANOVA. 


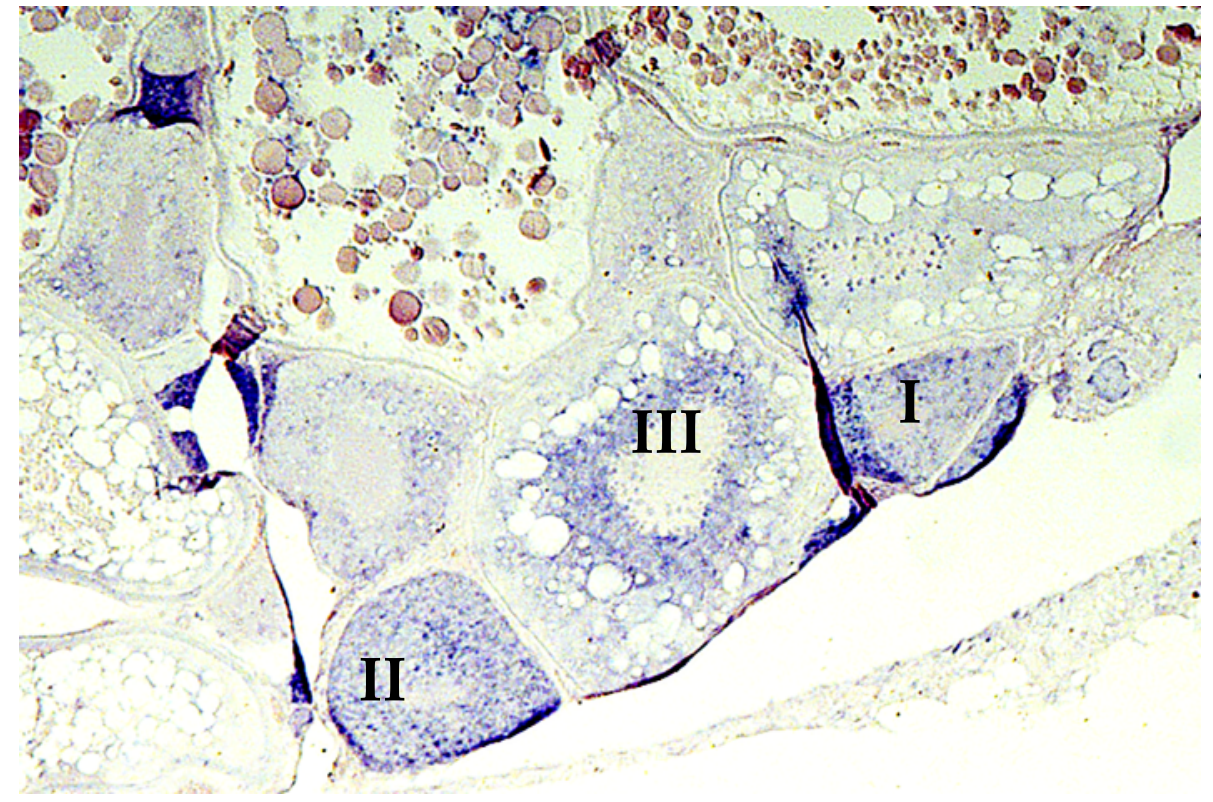

Supplementary Figure 6. in situ hybridization of stag2a in adult zebrafish ovary. Expression of stag2a as detected by in situ hybridization (blue/purple) is clearly visible in Stage I, II and III oocytes in this transverse section through wild type adult zebrafish ovary. 
bioRxiv preprint doi: https://doi.org/10.1101/2020.10.19.346122; this version posted October 19, 2020. The copyright holder for this preprint (which was not certified by peer review) is the author/funder, who has granted bioRxiv a license to display the preprint in perpetuity. It is made $\underline{\text { Supplementary Tables }}$ available under aCC-BY-NC-ND 4.0 International license.

Supplementary Table 1. List of accession identifiers for proteins used for phylogenetic analysis.

\begin{tabular}{cc}
\hline Protein & Accession ID \\
\hline Hs STAG1 & NP_005853.2 \\
\hline Hs STAG2 & NP_001036214.1 \\
\hline Gg STAG1 & XP_015146838.1 \\
\hline Gg STAG2 & XP_004940885.1 \\
\hline Mm Stag1 & NP_001344193.1 \\
\hline Mm Stag2 & NP_001071180.1 \\
\hline Xt stag1 & NP_001121432.1 \\
\hline Xt stag2 & XP_002931833.2 \\
\hline Dr stag1a & NP_001349269.1 \\
\hline Dr stag1b & XP_692120.3 \\
\hline Dr stag2a & NP_001093498.1 \\
\hline Dr stag2b & XP_005173250.1
\end{tabular}

Supplementary Table 2. sgRNA sequences used to generate CRISPR mutants. PAM sequences are marked in blue.

\begin{tabular}{ccc}
\hline Target gene & Sequence 5'-3' & CHOPCHOP in silico efficiency \\
\hline stag1 $a$ & GGGCTTTATGGCAGTCCAGAGGG & 49.5 \\
\hline stag1 $b$ & CGGGAGGAGGCCGAATGGAGTGG & 54.11 \\
\hline stag $2 b$ & GGCCCTGGAGAGAAGGGAAAAGG & 45.19 \\
\hline
\end{tabular}

Supplementary Table 3. Primer sequences used in this study.

\begin{tabular}{ccc}
\hline Target gene & Forward primer & Reverse primer \\
\hline & Primers used for qPCR & \\
\hline stag1 $a$ & CTGGACCTTACATGACCGGC & TATCCAGCGTCATGGACACG \\
\hline stag1 $b$ & CCAGGTTGATGCAGAAAAGGTG & GGCGTCCAGATGCTTTTCCAT \\
\hline stag $2 a$ & AGCCGCTTCAAGGATCGAAT & CAGCGTCAGCAGCTTAATGG \\
\hline stag $2 b$ & CAATAGCAGAGATCCGGGCG & GACACTTCAGACGCACCTCA \\
\hline gata1 $a$ & TTACTGCCACCCGTTGATGT & TTGGCGAACTGGACTGTGTC \\
\hline & Primers used for in situ hybridisation & \\
\hline stag1 $a$ & CTTTGCCCTCACCTTCGGAT & GAGTTCTGCTCTCTCTCGCC \\
\hline stag1 $b$ & GTCTGAAGCATTCTGGGGCT & GGCATCCCTGTAACGGTGAA \\
\hline stag $2 a$ & AAGGGCGAAATGGCAAACTT & GACGCACCTCACCTTGCTTA \\
\hline stag $2 b$ & CATCCTCACTGTTGGCCTGT & GACACTTCAGACGCACCTCA \\
\hline & Primers used for genotyping & \\
\hline stag1 $a$ CRISPR & GCCTCGGAAGTCTCCATCAG & GCACACCTGCATAGCACTCT \\
\hline stag1 $b$ CRISPR & GGCGGCTAATAAGAAGGCCA & AAGCAGCACACAACCTCGAA \\
\hline stag $2 b$ CRISPR & GTCACTCTGCTTCAGGCGAA & TGACCTGCATGGCACTCTTC \\
\hline
\end{tabular}


bioRxiv preprint doi: https://doi.org/10.1101/2020.10.19.346122; this version posted October 19, 2020. The copyright holder for this preprint (which was not certified by peer review) is the author/funder, who has granted bioRxiv a license to display the preprint in perpetuity. It is made

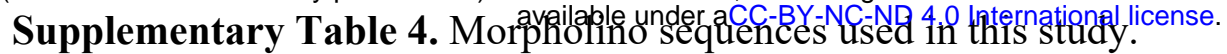

\begin{tabular}{ccc}
\hline Name & Sequence 5'-3' & Binding site \\
\hline stag1a & GGTTAGATGTTGTGTTACAGGTCT & 5'UTR \\
\hline stag $2 b$ & GTAATTCCGGTGCGGCTATCATTTC & ATG \\
\hline
\end{tabular}

